\title{
Phase field modeling and simulation of three-phase flows
}

\author{
JUNSEOK KIM ${ }^{\dagger}$ AND JOHN LOWENGRUB \\ Department of Mathematics, University of California, \\ 103 Multipurpose Science and Technology Building, Irvine, CA 92697-3875, USA
}

[Received 15 November 2004 and in revised form 5 June 2005]

\begin{abstract}
We derive a thermodynamically consistent phase-field model for flows containing three (or more) liquid components. The model is based on a Navier-Stokes (NS) and Cahn-Hilliard system (CH) which accounts for surface tension among the different components and three-phase contact lines. We develop a stable conservative, second order accurate fully implicit discretization of the NS and threephase (ternary) $\mathrm{CH}$ system. We use a nonlinear multigrid method to efficiently solve the discrete ternary $\mathrm{CH}$ system at the implicit time-level and then couple it to a multigrid/projection method that is used to solve the NS equation. We demonstrate convergence of our scheme numerically and perform numerical simulations to show the accuracy, flexibility, and robustness of this approach. In particular, we simulate a three-interface contact angle resulting from a spreading liquid lens on an interface, a buoyancy-driven compound drop, and the Rayleigh-Taylor instability of a flow with three partially miscible components.
\end{abstract}

Keywords: Ternary Cahn-Hilliard system; nonlinear multigrid; ternary fluid flow; interfacial tension; arbitrary miscibility.

\section{Introduction}

Many biomedical, chemical, and industrial processes involve mixtures of three or more liquid components. In spite of the importance of three-phase flows, most studies of three-phase systems do not consider hydrodynamical interactions (e.g. see [4-7, 10, 15, 20, 22]). There have been few theoretical and numerical studies of flows containing three or more liquid components compared to the large body of research for two-phase fluid flows. This is partly due to the difficulties in dealing with hydrodynamics associated with interfaces and triple junctions. In [40], a projection method is used for the motion of a triple junction in level set framework. In this approach, a linear projection of the level set functions onto a reduced set of variables is used to solve problems at triple lines and other multiple junctions (i.e., quadruple points). In [28], a compound drop consisting of three immiscible fluids is simulated using the immersed boundary method [37]. The presence of the interface and its effect on the flow is established via source terms in the governing equations.

There are a number of three-phase models that describe two immiscible fluids and surfactant. In low Reynolds number flows, boundary integral methods have been used to study the effect of surfactants on drop dynamics (e.g. [33, 35, 41]) and tip-streaming [19]. In [18, 24, 39], the effect of insoluble surfactants on drop deformation is studied in two and three dimensions using volume-of-fluid methods. In [25] the effect of surfactants on the dynamics of rising bubbles is

\footnotetext{
${ }^{\dagger}$ E-mail: jskim@math.uci.edu

‡E-mail: lowengrb@math.uci.edu
} 
investigated using an immersed boundary/front-tracking algorithm. In [13], a hybrid level-set/fronttracking algorithm was used to study the effect of surfactants on capillary waves. Further, in [27], the effect of surfactants on the evolution of the shape of an initially nonspherical drop translating in an otherwise quiescent fluid at low Reynolds number is examined. A combination of the boundaryintegral method and a finite-difference scheme is used to solve the coupled fluid dynamics and surfactant transport problems.

In this paper, we build upon our results for two-phase hydrodynamic systems [30] and threephase systems in the absence of hydrodynamic interactions [31] to model and simulate general threephase hydrodynamic systems. Advantages of this approach over level-set and immersed boundary approaches described above, for immiscible three-phase systems, are: (1) we do not need to perform any correction steps to multiple junctions; (2) it is easy to incorporate other physical properties such as miscible and immiscible fluid components.

We derive a thermodynamically consistent system of governing equations based on a phase-field approach. The system of equations couples the Navier-Stokes equations for the fluid motion to a system of Cahn-Hilliard type (fourth order nonlinear advection-diffusion equations) for the phase variables. Here the phase fields have a definite physical meaning: they are the mass ratios of the fluid components.

We develop a conservative, second order accurate fully implicit discretization of the NS and three-phase (ternary) $\mathrm{CH}$ system. It can be shown that if the time step is small enough, the discretization has a discrete energy functional that is the natural discretization of the free energy on the continuous level. In practice, we find that the discrete energy is always nonincreasing. We use a nonlinear multigrid method to efficiently solve the discrete ternary $\mathrm{CH}$ system at the implicit time-level and then couple it to a multigrid/projection method that is used to solve the NS equation.

We present examples of flows with miscible and immiscible components. We demonstrate the convergence of our algorithm through a resolution study. In addition, we find good agreement with the theory for an equilibrium liquid lens (lying atop an interface). We provide demonstrations of liquid/liquid remediation. In the first example, a compound drop is simulated, in which a light fluid encapsulated a heavy contaminant drop. The light fluid causes the compound drop to rise and deposit the contaminant at an interface where it may be removed. In the second example, we investigate the diffusional transfer of a preferentially miscible contaminant from one immiscible phase to another. In this example, the transfer is enhanced by the flow and in particular the RayleighTaylor instability.

The contents of this paper are as follows. In Section 2, the governing equations are derived. In Section 3, we derive the discrete scheme and numerical solution. We also present the approximate projection method used to solve the discrete generalized NS equations. Numerical experiments are presented in Section 4 In Section 5 , conclusions are drawn.

\section{Derivation of the governing equations}

We begin by deriving a thermodynamically consistent system of governing equations for a general heterogeneous, isothermal mixture of $N_{f}$ fluids following the strategy developed for binary (twocomponent) fluids by Lowengrub and Truskinovksy [34]. Let the mass concentrations be $c_{k}=$ $M_{k} / M$ for $k=1, \ldots, N_{f}$, where $M_{k}$ are the masses of the components in a representative material volume $V$, and $M$ is the total mass of the mixture. Since $M=\sum_{k=1}^{N_{f}} M_{k}$, we have $\sum_{k=1}^{N_{f}} c_{k}=1$. Suppose that each component moves with a velocity $\mathbf{u}_{k}$ and has density $\rho_{k}=M_{k} / V_{k}$ where $V_{k}$ is 
the volume of fluid $k$. Introducing volume fraction $\phi_{k}=V_{k} / V$, we have the relation $\rho c_{k}=\rho_{k} \phi_{k}$ where $\rho=\sum_{k=1}^{N_{f}} \rho_{k} \phi_{k}$ is the density of the mixture. Herein, we will assume that each component is incompressible, i.e. $\rho_{k}$ is constant. Note that this does not mean that the mixture density $\rho$ is constant since $\rho$ depends on $\phi_{k}$ or, equivalently, on $c_{k}$. The relation between $\rho$ and $c_{i}$ is given by

$$
\frac{1}{\rho}=\sum_{k=1}^{N_{f}} \frac{c_{k}}{\rho_{k}}
$$

Such mixtures were termed quasi-incompressible by Lowengrub and Truskinovsky [34].

\subsection{Balance equations}

The balance of mass for each component is

$$
\frac{\partial\left(\rho_{k} \phi_{k}\right)}{\partial t}+\nabla \cdot\left(\rho_{k} \phi_{k} \mathbf{u}_{k}\right)=0
$$

Define the mass-averaged (mixture) velocity field

$$
\mathbf{u}=\frac{1}{\rho} \sum_{k=1}^{N_{f}} \phi_{k} \rho_{k} \mathbf{u}_{k}=\sum_{k=1}^{N_{f}} c_{k} \mathbf{u}_{k} ;
$$

then summing 2.2 in $k$ we obtain the balance of mass for the mixture

$$
\frac{\partial \rho}{\partial t}+\nabla \cdot(\rho \mathbf{u})=0
$$

From (2.2) and 2.4, we obtain the mass concentration equation:

$$
\rho \dot{c}_{k}=\nabla \cdot \mathbf{J}_{k},
$$

where ${ }^{\circ}=\partial_{t}+\mathbf{u} \cdot \nabla$ is the advective derivative with respect to the mixture velocity, and

$$
\mathbf{J}_{k}=-\rho c_{k} \mathbf{w}_{k}, \quad \text { where } \quad \mathbf{w}_{k}=\left(\mathbf{u}_{k}-\mathbf{u}\right),
$$

is the diffusion flux.

For each component, we have the balance of linear momentum

$$
\rho c_{k} \frac{D^{k} \mathbf{u}_{k}}{D t}=\nabla \cdot \mathbf{P}_{k}+\rho c_{k} \mathbf{g}+\pi_{k},
$$

where $D^{k} / D t=\partial_{t}+\mathbf{u}_{k} \cdot \nabla$ is the advective derivative with respect to the component velocity, $\mathbf{P}_{k}$ is the stress tensor, $\mathbf{g}$ is the gravity force and $\pi_{k}$ are the forces per unit volume due to interactions with other phases. Note that as yet $\mathbf{P}_{k}$ and $\pi_{k}$ are unspecified. Summing over $k$, and requiring that $\sum_{k=1}^{N_{f}} \pi_{k}=0$, which is necessary for the conservation of linear momentum of the mixture, we get the following linear momentum equation for the mixture:

$$
\rho \dot{\mathbf{u}}=\nabla \cdot \mathbf{P}+\rho \mathbf{g},
$$

where $\mathbf{P}=\sum_{k=1}^{N_{f}}\left(\mathbf{P}_{k}-\rho c_{k} \mathbf{w}_{k} \otimes \mathbf{w}_{k}\right)$ is the stress tensor of the mixture. 
Following classical theory (e.g. see [17] and Lowengrub and Truskinovsky [34]), we focus on the mixture equations and not on the detailed force interactions (e.g. $\left.\pi_{i}\right)$. Therefore, we consider an energy balance for the entire system and derive thermodynamically consistent constitutive relations for $\mathbf{P}$ and $\mathbf{J}_{k}$ as follows. Let $\Omega$ be an arbitrary domain that moves with the mixture velocity $\mathbf{u}$. Then the integral form of the energy balance for the mixture is

$$
\frac{\mathrm{d}}{\mathrm{d} t} \int_{\Omega(t)}\left(\rho e+\frac{1}{2} \rho|\mathbf{u}|^{2}\right) \mathrm{d} \Omega=\int_{\partial \Omega}\left(\mathbf{P n} \cdot \mathbf{u}+\sum_{k=1}^{N_{f}}\left(\mathbf{t}_{k} \cdot \mathbf{n}\right) \dot{c}_{k}\right) \mathrm{d} \partial \Omega+\int_{\Omega}(r+\rho \mathbf{g} \cdot \mathbf{u}) \mathrm{d} \Omega,
$$

where $e$ is the internal energy, $\rho|\mathbf{u}|^{2} / 2$ is the kinetic energy and $\mathbf{n}$ is the outward normal vector to $\partial \Omega$. The first term on the right hand side is the rate of work done on $\partial \Omega$ by the fluid stress and the extra stresses due to concentration gradients, i.e. $\mathbf{t}_{k}$ is a generalized force that is as yet undetermined. This term is suggested by the variational analysis of Lowengrub and Truskinovsky [34]. In the second term on the RHS, $r$ is the density of heat sources necessary to ensure that the temperature is constant and the remaining term is the rate of work done due to gravity.

Using the mixture mass (2.4) and momentum balance equations (2.8), the local form of the energy balance equation 2.9 is

$$
\rho \dot{e}=\mathbf{P}: \nabla \mathbf{u}+\sum_{k=1}^{N_{f}} \nabla \cdot\left(\mathbf{t}_{k} \dot{c}_{k}\right)+r
$$

\subsection{Thermodynamics and constitutive relations}

Because we are dealing with isothermal flow, it is useful to introduce the Helmholtz free energy $\mathcal{F}$ rather than the internal energy. The relation between the two is

$$
\mathcal{F}=e-T s,
$$

where $T$ is the temperature. Thus, 2.10 becomes

$$
\rho T \dot{s}=-\rho \dot{\mathcal{F}}+\mathbf{P}: \nabla \mathbf{u}+\sum_{i=1}^{N_{f}} \nabla \cdot\left(\mathbf{t}_{i} \dot{c}_{i}\right)+r .
$$

Next, we make the constitutive assumption that the free energy

$$
\mathcal{F}=\mathcal{F}\left(c_{1}, \ldots, c_{N_{f}}, \nabla c_{1}, \ldots, \nabla c_{N_{f}}\right)
$$

is such that

$$
\dot{\mathcal{F}}=\sum_{k=1}^{N_{f}}\left(\frac{\partial \mathcal{F}}{\partial c_{k}} \dot{c}_{k}+\frac{\partial \mathcal{F}}{\partial \nabla c_{k}}\left(\nabla c_{k}\right)^{\cdot}\right)
$$

Using the identity $\left(\nabla c_{k}\right)^{\cdot}=\nabla \dot{c}_{k}-(\nabla \mathbf{u})^{T} \cdot \nabla c_{k}$ (e.g. see [34]) and plugging 2.14 in 2.12 gives

$$
\begin{aligned}
\rho T \dot{s}= & \left(\mathbf{P}+\rho \sum_{k=1}^{N_{f}} \nabla c_{k} \otimes \frac{\partial \mathcal{F}}{\partial \nabla c_{k}}\right): \nabla \mathbf{u} \\
& +\sum_{k=1}^{N_{f}}\left(\mathbf{t}_{k}-\rho \frac{\partial \mathcal{F}}{\partial \nabla c_{k}}\right) \cdot \nabla \dot{c}_{k}-\rho \sum_{k=1}^{N_{f}}\left(\frac{\partial \mathcal{F}}{\partial c_{k}}-\frac{1}{\rho} \nabla \cdot \mathbf{t}_{k}\right) \dot{c}_{k}+r .
\end{aligned}
$$


Next observe that because the fluid components are incompressible the velocity gradient $\nabla \mathbf{u}$ and $\dot{c}_{k}$ are not independent. They are related via $(2.1)$ and 2.5 . That is, there is a degeneracy in 2.15 since

$$
\nabla \cdot \mathbf{u}=-\sum_{k=1}^{N_{f}} \frac{1}{\rho} \frac{\partial \rho}{\partial c_{k}} \dot{c}_{k}=-\sum_{k=1}^{N_{f}} \frac{1}{\rho^{2}} \frac{\partial \rho}{\partial c_{k}} \nabla \cdot \mathbf{J}_{k}
$$

Note that from (2.1) we see that

$$
-\frac{1}{\rho^{2}} \frac{\partial \rho}{\partial c_{k}}=\alpha_{k} \equiv \frac{1}{\rho_{k}}, \quad \text { for } k=1, \ldots N_{f},
$$

is a constant.

To exploit the degeneracy introduce a scalar Lagrange multiplier $p$. This is the mixture pressure. Then

$$
p \mathbf{I}: \nabla \mathbf{u}=\sum_{k=1}^{N_{f}} \alpha_{k} p \rho \dot{c}_{k},
$$

where $\mathbf{I}$ is the identity matrix. Using 2.18$)$ in 2.15, , we get

$$
\begin{aligned}
\rho T \dot{s}= & \left(\mathbf{P}+p \mathbf{I}+\rho \sum_{k=1}^{N_{f}} \nabla c_{k} \otimes \frac{\partial \mathcal{F}}{\partial \nabla c_{k}}\right): \nabla \mathbf{u} \\
& +\sum_{k=1}^{N_{f}}\left(\mathbf{t}_{k}-\rho \frac{\partial \mathcal{F}}{\partial \nabla c_{k}}\right) \cdot \nabla \dot{c}_{k}-\rho \sum_{k=1}^{N_{f}}\left(\frac{\partial \mathcal{F}}{\partial c_{k}}+\alpha_{k} p-\frac{1}{\rho} \nabla \cdot \mathbf{t}_{k}\right) \dot{c}_{k}+r .
\end{aligned}
$$

According to the second law of thermodynamics, in the form of the Clausius-Duhem inequality, we have

$$
\rho \chi \geqslant 0, \quad \text { where } \rho \chi \equiv \rho \dot{s}+\nabla \cdot \mathcal{J}-r / T,
$$

where $\chi$ is the internal dissipation and $\mathcal{J}$ is the entropy flux (see Truesdell and Noll [45]). Now, from (2.19) together with 2.5), we obtain

$$
\begin{aligned}
\rho \chi= & \frac{1}{T}\left(\mathbf{P}+p \mathbf{I}+\rho \sum_{k=1}^{N_{f}} \nabla c_{k} \otimes \frac{\partial \mathcal{F}}{\partial \nabla c_{k}}\right): \nabla \mathbf{u}+\frac{1}{T} \sum_{k=1}^{N_{f}}\left(\mathbf{t}_{k}-\rho \frac{\partial \mathcal{F}}{\partial \nabla c_{k}}\right) \cdot \nabla\left(\rho^{-1} \nabla \cdot \mathbf{J}_{k}\right) \\
& +\frac{1}{T} \sum_{k=1}^{N_{f}} \nabla \mu_{k} \cdot \mathbf{J}_{k}+\nabla \cdot\left(\mathcal{J}-\sum_{k=1}^{N_{f}} \frac{\mu_{k} J_{k}}{T}\right),
\end{aligned}
$$

where $\mu_{k}$ is the generalized chemical potential given by

$$
\mu_{k}=\frac{\partial \mathcal{F}}{\partial c_{k}}+\alpha_{k} p-\frac{1}{\rho} \nabla \cdot \mathbf{t}_{k}
$$

Next, because $\sum_{k=1}^{N_{f}} \mathbf{J}_{k}=0$ this leads to additional constraints on the constitutive relations for the stress tensor $\mathbf{P}$, the forces $\mathbf{t}_{k}$ and the fluxes $\mathbf{J}_{k}$. Since $\sum_{k=1}^{N_{f}} c_{k}=1$, the concentration fields are not 
independent. We may thus define $c_{N_{f}}=1-\sum_{k=1}^{N_{f}-1} c_{k}$. This leads to the equivalent entropy form

$$
\begin{aligned}
\rho \chi= & \frac{1}{T}\left(\mathbf{P}+p \mathbf{I}+\rho \sum_{k=1}^{N_{f}} \nabla c_{k} \otimes\left(\frac{\partial \mathcal{F}}{\partial \nabla c_{k}}-\frac{\partial \mathcal{F}}{\partial \nabla c_{N_{f}}}\right)\right): \nabla \mathbf{u} \\
& +\frac{1}{T} \sum_{k=1}^{N_{f}}\left(\mathbf{t}_{k}-\rho\left(\frac{\partial \mathcal{F}}{\partial \nabla c_{k}}-\frac{\partial \mathcal{F}}{\partial \nabla c_{N_{f}}}\right)\right) \cdot \nabla\left(\rho^{-1} \nabla \cdot \mathbf{J}_{k}\right) \\
& +\frac{1}{T} \sum_{k=1}^{N_{f}} \nabla\left(\mu_{k}-\mu_{N_{f}}\right) \cdot \mathbf{J}_{k}+\nabla \cdot\left(\mathcal{J}-\sum_{k=1}^{N_{f}} \frac{\left(\mu_{k}-\mu_{N_{f}}\right) J_{k}}{T}\right) .
\end{aligned}
$$

Taking the diffusion flux $\mathcal{J}=\sum_{k=1}^{N_{f}}\left(\mu_{k}-\mu_{N_{f}}\right) \mathbf{J}_{k} / T$ we are now in a position to pose thermodynamically consistent constitutive relations for $\mathbf{P}, \mathbf{t}_{k}$ and $\mathbf{J}_{k}$. Following Coleman and Noll [14] where $\nabla \mathbf{u}$ is varied independently from the other quantities leads to the constitutive assumptions

$$
\begin{aligned}
& \mathbf{P}=-p \mathbf{I}-\rho \sum_{k=1}^{N_{f}} \nabla c_{k} \otimes\left(\frac{\partial \mathcal{F}}{\partial \nabla c_{k}}-\frac{\partial \mathcal{F}}{\partial \nabla c_{N_{f}}}\right)+\eta\left(\mathbf{D}-\frac{2}{3}(\nabla \cdot \mathbf{u}) \mathbf{I}\right), \\
& \mathbf{t}_{k}=\rho\left(\frac{\partial \mathcal{F}}{\partial \nabla c_{k}}-\frac{\partial \mathcal{F}}{\partial \nabla c_{N_{f}}}\right), \\
& J_{k}=v_{k} \nabla\left(\mu_{k}-\mu_{N_{f}}\right), \text { for } k<N_{f}, \text { and } J_{N_{f}}=-\sum_{k=1}^{N_{f}-1} J_{k},
\end{aligned}
$$

where $\mathbf{D}=\nabla \mathbf{u}+\nabla \mathbf{u}^{T}$ is the rate of strain tensor and $\eta$ is the viscosity (note that the bulk viscosity is assumed to be 0 for simplicity and that $\eta$ may be a function of $c$ ). We thus obtain

$$
\rho \chi=\frac{\eta}{T} \mathbf{D}: \mathbf{D}+\frac{1}{T} \sum_{k=1}^{N_{f}} v_{k}\left|\nabla\left(\mu_{k}-\mu_{N_{f}}\right)\right|^{2} \geqslant 0
$$

and so the second law of thermodynamics is satisfied. Note that concentration gradients give rise to extra fluid stresses. As will be discussed later, these mimic surface tension stresses. Finally, although we have singled out one of the components, we demonstrate later that the evolution of our system does not depend on the labeling of the components. We also note that other constitutive relations may be taken (e.g. see Appendix A as well as [21]). Our choice here is consistent with that considered previously by Morral \& Cahn [36] for linearized three-phase systems.

\subsection{Summary of general equations}

Putting together the results from the previous section, the thermodynamically consistent system of equations governing a mixture of $N_{f}$ fluids is

$$
\nabla \cdot \mathbf{u}=\sum_{k=1}^{N_{f}}\left(\alpha_{k}-\alpha_{N_{f}}\right) \nabla \cdot\left(v_{k} \nabla\left(\mu_{k}-\mu_{N_{f}}\right)\right),
$$




$$
\begin{aligned}
\rho \dot{\mathbf{u}}= & -\nabla p-\nabla \cdot\left(\rho \sum_{k=1}^{N_{f}} \nabla c_{k} \otimes\left(\frac{\partial \mathcal{F}}{\partial \nabla c_{k}}-\frac{\partial \mathcal{F}}{\partial \nabla c_{N_{f}}}\right)\right) \\
& +\nabla \cdot\left(\eta(c)\left(\mathbf{D}-\frac{2}{3}(\nabla \cdot \mathbf{u}) \mathbf{I}\right)\right)+\rho \mathbf{g}, \\
\rho \dot{c}_{k}= & \nabla \cdot\left(v_{k} \nabla\left(\mu_{k}-\mu_{N_{f}}\right)\right),
\end{aligned}
$$

and

$$
\mu_{k}=\frac{\partial \mathcal{F}}{\partial c_{k}}+\alpha_{k} p-\frac{1}{\rho} \nabla \cdot\left(\rho\left(\frac{\partial \mathcal{F}}{\partial \nabla c_{k}}-\frac{\partial \mathcal{F}}{\partial \nabla c_{N_{f}}}\right)\right), \quad \alpha_{k}=\frac{1}{\rho_{k}},
$$

for $k=1, \ldots, N_{f}-1$ and $c_{N_{f}}=1-\sum_{k=1}^{N_{f}-1} c_{k}$. This system couples a generalized Navier-Stokes equation with a nonlinear advection-diffusion equation for the concentration.

\subsection{Special choice of free energy, the Navier-Stokes-Cahn-Hilliard system and nondimensionalization}

To make further progress, we need to choose the form of the Helmholtz free energy. Following Cahn and Hilliard [12], we take

$$
\mathcal{F}=F\left(c_{1}, \ldots, c_{N_{f}}\right)+\sum_{k=1}^{N_{f}} \frac{\epsilon_{k}^{2}}{4}\left|\nabla c_{k}\right|^{2}
$$

This gives

$$
\frac{\partial \mathcal{F}}{\partial \nabla c_{k}}=\frac{1}{2} \epsilon_{k}^{2} \nabla c_{k}
$$

Note that this makes the concentration equation $(2.30)$ a fourth order nonlinear advection-diffusion equation and is a generalization of the classical Cahn-Hilliard equation used to describe phase separation in binary mixtures [12]. Further, the extra stress in the Navier-Stokes equation 2.29] can be written as

$$
\rho \sum_{k=1}^{N_{f}} \nabla c_{k} \otimes\left(\frac{\partial \mathcal{F}}{\partial \nabla c_{k}}-\frac{\partial \mathcal{F}}{\partial \nabla c_{N_{f}}}\right)=\rho \sum_{k=1}^{N_{f}} \frac{\epsilon_{k}^{2}}{2} \nabla c_{k} \otimes \nabla c_{k}
$$

where we have used the fact that $\sum_{k=1}^{N_{f}} c_{k}=1$. Further, we have

$$
\mu_{k}-\mu_{N_{f}}=\frac{\partial F}{\partial c_{k}}-\frac{\partial F}{\partial c_{N_{f}}}+\left(\alpha_{k}-\alpha_{N_{f}}\right) p-\frac{1}{2 \rho} \nabla \cdot\left(\rho \nabla\left(\epsilon_{k}^{2} c_{k}+\epsilon_{N_{f}}^{2} \sum_{j=1}^{N_{f}-1} c_{j}\right)\right),
$$

where we have again used $\sum_{k=1}^{N_{f}} c_{k}=1$. We may therefore define, for $k=1, \ldots, N_{f}-1$,

$$
\begin{aligned}
\tilde{F}\left(c_{1}, \ldots, c_{N_{f}-1}\right) & =F\left(c_{1}, \ldots, c_{N_{f}-1}, 1-\sum_{k=1}^{N_{f}-1} c_{k}\right), \\
\tilde{\alpha}_{k} & =\alpha_{k}-\alpha_{N_{k}},
\end{aligned}
$$


so that

$$
\frac{\partial \tilde{F}}{\partial c_{k}}=\frac{\partial F}{\partial c_{k}}-\frac{\partial F}{\partial c_{N_{f}}}
$$

Analogously, one may define

$$
\begin{aligned}
\tilde{\mu}_{k} & =\mu_{k}-\mu_{N_{f}} \\
& =\frac{\partial \tilde{F}}{\partial c_{k}}+\tilde{\alpha}_{k} p-\frac{1}{2 \rho} \nabla \cdot\left(\rho \nabla\left(\epsilon_{k}^{2} c_{k}+\epsilon_{N_{f}}^{2} \sum_{j=1}^{N_{f}-1} c_{j}\right)\right)
\end{aligned}
$$

for $k=1, \ldots, N_{f}-1$. The resulting system is a coupled Navier-Stokes and Cahn-Hilliard system (see also [34]). We note that by equipping this system with natural boundary conditions, i.e. $\mathbf{u}=\mathbf{0}=$ $\nabla c_{k} \cdot \mathbf{n}$ and $\nabla \tilde{\mu}_{k} \cdot \mathbf{n}=0$, this system (with gravity $\mathbf{g}=\mathbf{0}$ ) has a discrete (free) energy functional

$$
E(t)=\int_{\Omega}\left(\rho\left(\tilde{F}\left(c_{1}, \ldots, c_{N_{f}-1}\right)+\sum_{k=1}^{N_{f}} \frac{\epsilon_{k}^{2}}{4}\left|\nabla c_{k}\right|^{2}\right)+\frac{\rho}{2}|\mathbf{u}|^{2}\right) \mathrm{d} \Omega,
$$

where $\Omega$ is the (fixed) physical domain. In the remainder of the paper, we drop the tilde notation for simplicity.

We nondimensionalize the system as follows (e.g. see also [34]). Let $L_{*}$ and $V_{*}$ denote characteristic scales of length and velocity. Then introduce the dimensionless independent variables $\bar{x}=x / L$ and $\bar{t}=V_{*} t / L_{*}$ and the natural scaling of the dependent variables $\overline{\mathbf{u}}=\mathbf{u} / V_{*}, \bar{\rho}=\rho / \rho_{*}$, $\bar{\eta}=\eta / \eta_{*}, \bar{p}=p /\left(\rho_{*} V_{*}^{2}\right), \bar{\mu}_{k}=\mu_{k} / \mu_{k}^{*}$, etc., where again the stars denote characteristic quantities. We also assume that the Helmholtz free energy is given by the sum

$$
F=\sum_{k=1}^{N_{f}} \mu_{k}^{*} \bar{F}_{k}
$$

The flow is then governed by the following nondimensional parameters:

$$
\begin{aligned}
& \mathcal{C}_{k}=\frac{\epsilon_{k}}{L_{*} \sqrt{\mu_{k}^{*}}}, \quad M_{k}=\frac{\sigma_{k} / \rho_{*}}{L_{*} \mu_{k}^{*}}, \operatorname{Pe}_{k}=\frac{\rho_{*} V_{*} L_{*}}{\nu_{k}^{*} \mu_{k}^{*}}, \\
& \mathcal{A}_{k}=\rho^{*} \alpha_{k}, \quad \mathrm{We}_{k}=\frac{\rho_{*} L_{*} V_{*}^{2}}{\sigma_{k}}, \quad \operatorname{Re}=\frac{\rho_{*} V_{*} L_{*}}{\eta_{*}}, \quad \mathrm{Fr}=\frac{V_{*}}{\sqrt{L_{*}|\mathbf{g}|}},
\end{aligned}
$$

where the top row are the nonclassical additional parameters introduced in the model and the second row are the classical fluid dynamics parameters. In the top row, the first parameter $\mathcal{C}_{k}$ is the Cahn number that is a nondimensional measure of the interface energy of the $k$ th component, the second $M_{k}$ is a measure of the relative strength of the surface tension and chemical energies and the third $\mathrm{Pe}_{k}$ is the diffusional Peclet number that measures the relative strengths of (chemical) diffusion and advection. In the bottom row, the first parameter $\mathcal{A}_{k}$ is a nondimensional measure of the density differences between components, the second $\mathrm{We}_{k}$ is the Weber number where $\sigma_{k}$ is the phase specific surface tension (see [40] and below), the third Re is the Reynolds number that measures the relative strength of inertial and viscous forces, and finally the last Fr is the Froude number that measures the relative strengths of the inertial and gravitational forces. 
Omitting the bar notation, the nondimensional Navier-Stokes-Cahn-Hilliard (NSCH) system (2.28)-2.31) is written as

$$
\begin{aligned}
\nabla \cdot \mathbf{u}= & \sum_{k=1}^{N_{f}-1} \frac{\mathcal{A}_{k}}{\mathrm{Pe}_{k}} \nabla \cdot\left(v_{k} \nabla \mu_{k}\right), \\
\rho \dot{\mathbf{u}}= & -\nabla p-\nabla \cdot\left(\rho \sum_{k=1}^{N_{f}} \frac{\mathcal{C}_{k}^{2}}{M_{k} \mathrm{We}_{k}} \nabla c_{k} \otimes \nabla c_{k}\right) \\
& +\frac{1}{\operatorname{Re}} \nabla \cdot\left(\eta(c)\left(\mathbf{D}-\frac{2}{3}(\nabla \cdot \mathbf{u}) \mathbf{I}\right)\right)+\frac{\rho-1}{\mathrm{Fr}^{2}} \mathbf{G}, \\
\rho \dot{c}_{k}= & \frac{1}{\mathrm{Pe}_{k}} \nabla \cdot\left(v_{k} \nabla \mu_{k}\right),
\end{aligned}
$$

where we have subtracted a linear term $\rho_{*} \mathbf{x} \cdot \mathbf{g}$ from the pressure and $\mathbf{G}=\mathbf{g} /|\mathbf{g}|$. Further, the chemical potential is

$$
\mu_{k}=\frac{\partial \tilde{\tilde{F}}}{\partial c_{k}}+\mathcal{A}_{k} \mathrm{We}_{k} M_{k} p-\frac{1}{\rho} \nabla \cdot \rho\left(\frac{\mathcal{C}_{k}^{2}}{2} \nabla c_{k}+\frac{\mathcal{C}_{N_{f}}^{2}}{2} \sum_{i=1}^{N_{f}-1} \nabla c_{i}\right),
$$

for $k=1, \ldots, N_{f}-1$, where

$$
\frac{\partial \tilde{\tilde{F}}}{\partial c_{k}}=\sum_{j=1}^{N_{f}} \frac{\mu_{j}^{*}}{\mu_{k}^{*}} \frac{\partial \bar{F}_{j}}{\partial c_{k}} .
$$

Finally, the nondimensional total free energy is

$$
E(t)=\int_{\Omega} \frac{\rho}{2}|\mathbf{u}|^{2} \mathrm{~d} \Omega+\sum_{k=1}^{3} \frac{1}{M_{k} \mathrm{We}_{k}} \int_{\Omega} \rho\left(\bar{F}_{k}+\frac{\mathcal{C}_{k}^{2}}{4}\left|\nabla c_{k}\right|^{2}\right) \mathrm{d} \Omega .
$$

Hereafter, we drop the bar and double tilde notation.

\subsection{Asymptotics and the sharp interface regime}

The miscibility properties of the flow components can be described through the free energy $F\left(c_{1}, c_{2}\right)$. For example, if all the components are miscible the free energy is a convex function of its components (i.e. the Hessian matrix is positive definite). If the components are immiscible, the free energy is a nonconvex function of its components to reflect the coexistence of multiple phases. Various mixtures of miscible and immiscible components can analogously be described. When the flow components are immiscible, the NSCH system should reduce to the classical NavierStokes equations together with the Laplace-Young surface tension jump conditions across interfaces and multi-junctions. This requires assumptions on the nonclassical parameters $\mathcal{C}_{k}, M_{k}$ and $\operatorname{Pe}_{k}$. Following the asymptotic analyses of Lowengrub and Truskinovsky [34] and others (e.g. see the review [2]) leads to the scaling

$$
\mathcal{C}_{k}=\epsilon^{2}, \quad M_{k}=\epsilon / \beta \quad \text { and } \quad \operatorname{Pe}_{k}=O(1) \text { or } O(1 / \epsilon),
$$


where the parameter $\epsilon$ is a nondimensional measure of interface thickness. Then it can be shown that in the sharp interface limit $\epsilon \rightarrow 0$, the classical Navier-Stokes system equations and jump conditions are recovered. An interface separating two immiscible fluids has an equilibrium profile $c^{\mathrm{eq}}(z)$ where $z$ is the coordinate in the normal direction to the interface. The parameter $\beta$ is then given by

$$
\beta=\left(\int_{-\infty}^{+\infty} \rho\left(c^{\mathrm{eq}}\right)\left(\frac{\partial c^{\mathrm{eq}}}{\partial z}\right)^{2} \mathrm{~d} z\right)^{-1} .
$$

For example, with a free energy $F(c)=\frac{1}{4} c_{1}^{2} c_{2}^{2}$, where we assume that $c_{3}=0$, we have

$$
c^{\mathrm{eq}}(z)=(1-\tanh (z / 2 \epsilon \sqrt{2})) / 2
$$

and, if $\rho(c)=1$, then $\beta=6 \sqrt{2}$.

For three-phase flows, following [40], the surface tension $\sigma_{i j}$ between immiscible flow components $i$ and $j$ is decomposed into the phase-specific surface tensions $\sigma_{i}$ and $\sigma_{j}$ by

$$
\sigma_{i j}=\sigma_{i}+\sigma_{j}
$$

That is, given $\sigma_{i j}$, a linear system of equations is solved for $\sigma_{i}$ and $\sigma_{j}$. The phase-specific surface tensions are used in the definition of the Weber number 2.42. For flows containing four or more immiscible fluids, the decomposition above is overdetermined and it is possible that no solution exists. Nevertheless, the model system $2.43-(2.48)$ is still valid although an alternate means may need to be used to determine the individual Weber numbers $\mathrm{We}_{k}$. This is currently under study.

\subsection{The Boussinesq approximation and the ternary system}

We next consider the special case of a ternary system and use the Boussinesq approximation. In the Boussinesq approximation, the densities of the flow components are nearly matched such that $\rho \approx 1$ but the Froude number may be small enough such that $(\rho-1) / \operatorname{Fr}^{2}$ is nonnegligible. Thus, in 2.43 -2.46, we take $\rho=1$ except in the gravitational term and we take $\mathcal{A}_{k}=0$. The resulting system is the ternary version of model $\mathrm{H}$ in the nomenclature of Hohenberg and Halperin [23].

The composition of a ternary mixture (A, B, and $\mathrm{C}$ ) can be mapped onto an equilateral triangle (the Gibbs triangle [38]) whose corners represent $100 \%$ concentration of A, B or C as shown in Fig. 2.1 (a). Mixtures with components lying on lines parallel to $\overline{B C}$ contain the same percentage of $\mathrm{A}$, those with lines parallel to $\overline{A C}$ have the same percentage of $\mathrm{B}$ concentration, and analogously for the $\mathrm{C}$ concentration. In Fig. 2.1 (a), the mixture at the position marked 'o' contains $60 \% \mathrm{~A}, 10 \% \mathrm{~B}$, and $30 \% \mathrm{C}$ (the total percentage must sum to $100 \%$ ).

Let $\mathbf{c}=\left(c_{1}, c_{2}\right)$ be the phase variable (i.e. concentrations of component $\mathrm{A}$ and component $\mathrm{B}$ ). Since $c_{1}+c_{2}+c_{3}=1$ we only need to solve the equations with $c_{1}$ and $c_{2}$. Here, for simplicity, we consider a constant mobility $1{ }^{1}\left(v_{k} \equiv 1\right) . F(\mathbf{c})$ is the Helmholtz free energy which is defined on the Gibbs triangle. For three immiscible fluids, the free energy can be modeled by

$$
F(\mathbf{c})=\sum_{k=1}^{3} F_{k}, \quad F_{k}=c_{k}^{2}\left(c_{\mathrm{rem}(k, 3)+1}^{2}+c_{\mathrm{rem}(k+1,3)+1}^{2}\right) / 8
$$

\footnotetext{
1 The extension to more general $v_{k}=v_{k}\left(c_{1}, c_{2}\right)$ is straightforward.
} 


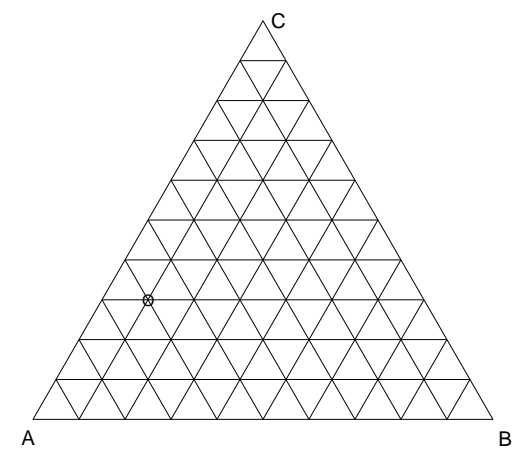

(a)

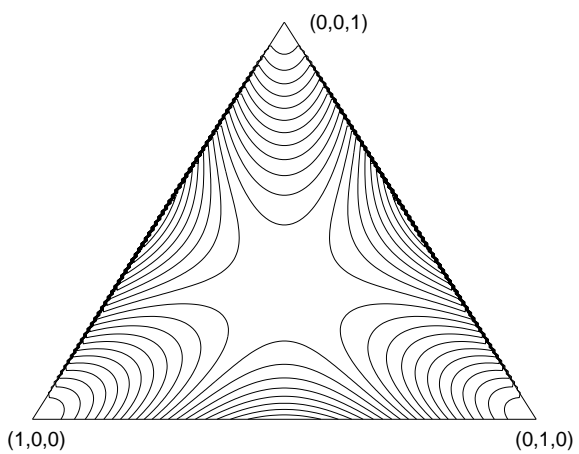

(b)

FIG. 2.1. (a) Gibbs triangle. (b) Contour plot of the free energy $F(\mathbf{c})$

where $\operatorname{rem}(x, y)$ is the remainder of the ratio $x / y$ and $c_{3}=1-c_{1}-c_{2}$. The contours of the free energy $F(\mathbf{c})$ projected onto the Gibbs triangle are shown in Fig. 2.1 (b). Note the energy minima are at the three vertices and the maximum is at the center.

The nondimensional Boussinesq ternary NSCH system is as follows:

$$
\begin{aligned}
\nabla \cdot \mathbf{u}= & 0, \\
\dot{\mathbf{u}}= & -\nabla p+\frac{1}{\mathrm{Re}} \nabla \cdot\left(\eta(\mathbf{c})\left(\nabla \mathbf{u}+\nabla \mathbf{u}^{T}\right)\right) \\
& -\sum_{k=1}^{3} \frac{\epsilon \beta}{\mathrm{We}_{k}} \nabla \cdot\left(\nabla c_{k} \otimes \nabla c_{k}\right)+\frac{\rho-1}{\mathrm{Fr}^{2}} \mathbf{g}, \\
\dot{\mathbf{c}}= & \frac{1}{\mathrm{Pe}} \Delta \boldsymbol{\mu}, \\
\boldsymbol{\mu}= & \mathbf{f}(\mathbf{c})-\Gamma_{\epsilon} \Delta \mathbf{c},
\end{aligned}
$$

where $\mathbf{f}(\mathbf{c})=\left(f_{1}(\mathbf{c}), f_{2}(\mathbf{c})\right)=\left(\partial_{c_{1}} F(\mathbf{c}), \partial_{c_{2}} F(\mathbf{c})\right)$ and $\Gamma_{\epsilon}$ is the matrix

$$
\Gamma_{\epsilon} \equiv\left(\begin{array}{cc}
\epsilon^{2} & \epsilon^{2} / 2 \\
\epsilon^{2} / 2 & \epsilon^{2}
\end{array}\right)
$$

The natural boundary and initial conditions for the ternary NSCH equation are

$$
\frac{\partial \mathbf{c}}{\partial n}=\frac{\partial \boldsymbol{\mu}}{\partial n}=0 \quad \text { and } \quad \mathbf{u}=\mathbf{0} \quad \text { on } \partial \Omega, \quad \mathbf{c}(\mathbf{x}, 0)=\mathbf{c}_{0}(\mathbf{x}), \quad \mathbf{u}(\mathbf{x}, 0)=\mathbf{0},
$$

where $\mathbf{n}$ is the normal unit vector pointing out of $\Omega$. Finally, the nondimensional total free energy for the Boussinesq system is

$$
E(t)=\int_{\Omega} \frac{1}{2}|\mathbf{u}|^{2} \mathrm{~d} \Omega+\sum_{k=1}^{3} \frac{1}{M_{k} \mathrm{We}_{k}} \int_{\Omega} \rho\left(F_{k}+\frac{\mathcal{C}_{k}^{2}}{4}\left|\nabla c_{k}\right|^{2}\right) \mathrm{d} \Omega,
$$

where $F=\sum_{k=1}^{3} F_{k}$. 


\section{Numerical solution}

The numerical solution of the ternary NSCH system uses a second-order accurate spatial discretization and a Crank-Nicholson type time stepping method. For simplicity and clarity of exposition, we will present the numerical method in 2D, but the extension to 3D is straightforward. The computational grid consists of square cells of a uniform size $h$; these cells $\Omega_{i j}$ are centered at $\left(x_{i}=(i-0.5) h, y_{j}=(j-0.5) h\right)$, where $i=1, \ldots, L$ and $j=1, \ldots, M$. Given $\mathbf{u}^{n-1}, \mathbf{u}^{n}$, $\mathbf{c}^{n-1}, \mathbf{c}^{n}$, defined at cell centers and $p^{n-1 / 2}$ defined at cell corners, we want to find $\mathbf{u}^{n+1}, \mathbf{c}^{n+1}$, and $p^{n+1 / 2}$. The outline of the algorithm is as follows:

Step 1. Initialize $\mathbf{c}^{0}$ to be the locally equilibrated concentration profile and $\mathbf{u}^{0}$ to be the divergencefree velocity field.

Step 2. Solve the $\mathrm{CH}$ system and update the concentration field $\mathbf{c}^{n}$ to $\mathbf{c}^{n+1}$. We use a nonlinear Full Approximation Storage (FAS) multigrid method to solve the nonlinear discrete system (3.1) and (3.2) given below at implicit time level. The nonlinearity is treated using a nonlinear GaussSeidel relaxation. Details of this step are presented in our recent paper [31]. Here, however, we have an additional source term due to advection. That is, we solve the following second order accurate discrete system:

$$
\begin{aligned}
\frac{\mathbf{c}_{i j}^{n+1}-\mathbf{c}_{i j}^{n}}{\Delta t} & =\frac{1}{\operatorname{Pe}} \Delta_{d} \boldsymbol{\mu}_{i j}^{n+1 / 2}-\left(\mathbf{u} \cdot \nabla_{d} \mathbf{c}\right)^{n+1 / 2}, \\
\boldsymbol{\mu}_{i j}^{n+1 / 2} & =\hat{\phi}\left(\mathbf{c}_{i j}^{n}, \mathbf{c}_{i j}^{n+1}\right)-\frac{1}{2} \Gamma_{\epsilon} \Delta_{d}\left(\mathbf{c}_{i j}^{n}+\mathbf{c}_{i j}^{n+1}\right),
\end{aligned}
$$

where $\Delta_{d}$ is the standard five-point discretization of the Laplacian operator in 2D. The advection term $\left(\mathbf{u} \cdot \nabla_{d} \mathbf{c}\right)^{n+1 / 2}$ is approximated by using a fifth order weighted essentially nonoscillatory (WENO) scheme [26] and is described in Sec. 3.1. We have $\hat{\phi}=\left(\hat{\phi}_{1}, \hat{\phi}_{2}\right)$ where $\hat{\phi}_{1}(\ldots)$ and $\hat{\phi}_{2}(\ldots)$ denote Taylor series approximations to $f_{1}$ and $f_{2}$ up to second order, respectively:

$$
\begin{aligned}
\hat{\phi}_{1}\left(\mathbf{c}^{n}, \mathbf{c}^{n+1}\right)= & f_{1}\left(\mathbf{c}^{n+1}\right)-\frac{1}{2} \partial_{c_{1}} f_{1}\left(\mathbf{c}^{n+1}\right)\left(c_{1}^{n+1}-c_{1}^{n}\right) \\
& -\frac{1}{2} \partial_{c_{2}} f_{1}\left(\mathbf{c}^{n+1}\right)\left(c_{2}^{n+1}-c_{2}^{n}\right)+\frac{1}{3 !} \partial_{c_{1}}^{2} f_{1}\left(\mathbf{c}^{n+1}\right)\left(c_{1}^{n+1}-c_{1}^{n}\right)^{2} \\
& +\frac{2}{3 !} \partial_{c_{2}} \partial_{c_{1}} f_{1}\left(\mathbf{c}^{n+1}\right)\left(c_{1}^{n+1}-c_{1}^{n}\right)\left(c_{2}^{n+1}-c_{2}^{n}\right)+\frac{1}{3 !} \partial_{c_{2}}^{2} f_{1}\left(\mathbf{c}^{n+1}\right)\left(c_{2}^{n+1}-c_{2}^{n}\right)^{2}
\end{aligned}
$$

and

$$
\begin{aligned}
\hat{\phi}_{2}\left(\mathbf{c}^{n}, \mathbf{c}^{n+1}\right)= & f_{2}\left(\mathbf{c}^{n+1}\right)-\frac{1}{2} \partial_{c_{1}} f_{2}\left(\mathbf{c}^{n+1}\right)\left(c_{1}^{n+1}-c_{1}^{n}\right) \\
& -\frac{1}{2} \partial_{c_{2}} f_{2}\left(\mathbf{c}^{n+1}\right)\left(c_{2}^{n+1}-c_{2}^{n}\right)+\frac{1}{3 !} \partial_{c_{1}}^{2} f_{2}\left(\mathbf{c}^{n+1}\right)\left(c_{1}^{n+1}-c_{1}^{n}\right)^{2} \\
& +\frac{2}{3 !} \partial_{c_{2}} \partial_{c_{1}} f_{2}\left(\mathbf{c}^{n+1}\right)\left(c_{1}^{n+1}-c_{1}^{n}\right)\left(c_{2}^{n+1}-c_{2}^{n}\right)+\frac{1}{3 !} \partial_{c_{2}}^{2} f_{2}\left(\mathbf{c}^{n+1}\right)\left(c_{2}^{n+1}-c_{2}^{n}\right)^{2} .
\end{aligned}
$$

As described in [31], this discretization ensures that in the absence of flow, a discrete version of equations 2.56-2.57) is nonincreasing in time independent of the choice of $\Delta t$. This gives enhanced stability. 
Step 3. Update the velocity $\mathbf{u}^{n}$ to $\mathbf{u}^{n+1}$ and the pressure $p^{n+1 / 2}$. We use the following approximate projection method adapted from [8]. We solve

$$
\begin{aligned}
\frac{\mathbf{u}^{*}-\mathbf{u}^{n}}{\Delta t}= & -\nabla_{d} p^{n-1 / 2}+\frac{1}{2 \operatorname{Re}} \nabla_{d} \cdot \eta\left(\mathbf{c}^{n+1}\right)\left[\nabla_{d} \mathbf{u}^{*}+\left(\nabla_{d} \mathbf{u}^{*}\right)^{T}\right]+\frac{\rho\left(\mathbf{c}^{n+1 / 2}\right)-1}{\mathrm{Fr}^{2}} \mathbf{G} \\
& +\frac{1}{2 \operatorname{Re}} \nabla_{d} \cdot \eta\left(\mathbf{c}^{n}\right)\left[\nabla_{d} \mathbf{u}^{n}+\left(\nabla_{d} \mathbf{u}^{n}\right)^{T}\right]+\mathbf{F}_{\mathrm{st}}^{n+1 / 2}-\left(\mathbf{u} \cdot \nabla_{d} \mathbf{u}\right)^{n+1 / 2}
\end{aligned}
$$

using a multigrid method for the intermediate velocity $\mathbf{u}^{*}$ without strictly enforcing the incompressibility constraint. We use $\mathbf{F}_{\mathrm{st}}=\sum_{k=1}^{3} \frac{\epsilon \beta}{\mathrm{We}_{k}} \nabla \cdot\left(\left|\nabla c_{k}\right|^{2} I-\nabla c_{k} \otimes \nabla c_{k}\right)$ and the pressure field is replaced by $p+\sum_{k=1}^{3} \frac{\epsilon \beta}{\mathrm{We}_{k}}\left|\nabla c_{k}\right|^{2}$. This modification is performed for two reasons. First, by taking into account analytic cancellation a simpler and more accurate discretization may be obtained. Second, when the interface is flat, this term is equal to zero, which is consistent with the fact that the interfacial force vanishes for flat interfaces.

The discretization of $\mathbf{F}_{\mathrm{st}}$ is given in Sec. 3.2. The terms $\nabla_{d} p$ and $\nabla_{d} \cdot \eta(\mathbf{c})\left[\nabla_{d} \mathbf{u}+\nabla_{d} \mathbf{u}^{T}\right]$ are defined as follows:

$$
\begin{aligned}
\left(\nabla_{d} p\right)_{i j}= & \left(\frac{p_{i+1 / 2, j+1 / 2}+p_{i+1 / 2, j-1 / 2}-p_{i-1 / 2, j+1 / 2}-p_{i-1 / 2, j-1 / 2}}{2 h},\right. \\
& \left.\frac{p_{i+1 / 2, j+1 / 2}-p_{i+1 / 2, j-1 / 2}+p_{i-1 / 2, j+1 / 2}-p_{i-1 / 2, j-1 / 2}}{2 h}\right) .
\end{aligned}
$$

The first component of the viscous term $\nabla_{d} \cdot \eta(\mathbf{c})\left[\nabla_{d} \mathbf{u}+\nabla_{d} \mathbf{u}^{T}\right]$ is discretized as

$$
\begin{aligned}
\left(\nabla_{d} \cdot \eta(\mathbf{c})\left[\nabla_{d} \mathbf{u}+\nabla_{d} \mathbf{u}^{T}\right]\right)_{i j}^{1}=\frac{1}{h^{2}}\left(2 \eta\left(\mathbf{c}_{i+1 / 2, j}\right)\left(u_{i+1, j}-u_{i j}\right)-2 \eta\left(\mathbf{c}_{i-1 / 2, j}\right)\left(u_{i j}-u_{i-1, j}\right)\right. \\
+\eta\left(\mathbf{c}_{i, j+1 / 2}\right)\left(u_{i, j+1}-u_{i j}+0.25\left(v_{i+1, j+1}-v_{i-1, j+1}+v_{i+1, j}-v_{i-1, j}\right)\right) \\
\left.-\eta\left(\mathbf{c}_{i, j-1 / 2}\right)\left(u_{i j}-u_{i, j-1}+0.25\left(v_{i+1, j}-v_{i-1, j}+v_{i+1, j-1}-v_{i-1, j-1}\right)\right)\right) .
\end{aligned}
$$

The second component of the viscous term is discretized in a similar manner. The term $\left(\mathbf{u} \cdot \nabla_{d} \mathbf{u}\right)^{n+1 / 2}$ is computed using a fifth order WENO scheme described in Sec. 3.1

Then project $\mathbf{u}^{*}$ onto the space of approximately divergence-free vector fields and get the velocity $\mathbf{u}^{n+1}$, i.e., $\mathbf{u}^{*}=\mathbf{u}^{n+1}+\Delta t \nabla_{d} \phi$, where $\phi$ satisfies $\Delta_{d} \phi=\nabla_{d} \cdot \frac{\mathbf{u}^{*}-\mathbf{u}^{n}}{\Delta t}$ with the Neumann boundary condition, $\partial \phi / \partial \mathbf{n}=0$ (see [1] for more general boundary conditions). Note $\nabla_{d} \cdot \mathbf{u}^{n+1} \approx 0$ (see [8]). Finally, update pressure by $p^{n+1 / 2}=p^{n-1 / 2}+\phi$. This completes one time step.

\subsection{Approximation of the advection terms}

In this section, we describe the discretization of the advection terms. The values $\mathbf{u}_{i j}^{n+1 / 2}$ and $\mathbf{c}_{i j}^{n+1 / 2}$ are calculated using a second order accurate extrapolation from previous values, i.e., $\mathbf{u}_{i j}^{n+1 / 2}=$ $\left(3 \mathbf{u}_{i j}^{n}-\mathbf{u}_{i j}^{n-1}\right) / 2$ and $\mathbf{c}_{i j}^{n+1 / 2}=\left(3 \mathbf{c}_{i j}^{n}-\mathbf{c}_{i j}^{n-1}\right) / 2$. From these cell centered values we obtain cell edged values by $\mathbf{u}_{i+1 / 2, j}^{n+1 / 2}=\left(\mathbf{u}_{i j}^{n+1 / 2}+\mathbf{u}_{i+1, j}^{n+1 / 2}\right) / 2$ and $\mathbf{u}_{i, j+1 / 2}^{n+1 / 2}=\left(\mathbf{u}_{i j}^{n+1 / 2}+\mathbf{u}_{i, j+1}^{n+1 / 2}\right) / 2$.

In general, the normal velocities $u_{i+1 / 2, j}^{n+1 / 2}$ and $v_{i, j+1 / 2}^{n+1 / 2}$ at the edges are not divergence-free. To reduce the overall error, we apply the MAC projection [43] before construction of the convective 
derivatives. The equation

$$
\Delta_{d} \phi=\nabla_{\mathrm{MAC}} \cdot \mathbf{u}^{n+1 / 2}
$$

is solved for a cell centered $\phi$, where

$$
\left(\nabla_{\mathrm{MAC}} \cdot \mathbf{u}^{n+1 / 2}\right)_{i j}=\frac{u_{i+1 / 2, j}^{n+1 / 2}-u_{i-1 / 2, j}^{n+1 / 2}}{h}+\frac{v_{i, j+1 / 2}^{n+1 / 2}-v_{i, j-1 / 2}^{n+1 / 2}}{h}
$$

with the Neumann boundary condition, $\partial \phi / \partial \mathbf{n}=0$. The resulting linear system (3.4) is solved using a multigrid method with Gauss-Seidel relaxation. Then the discrete divergence-free cell-edge velocities $\tilde{u}$ and $\tilde{v}$ are defined by

$$
\tilde{u}_{i+1 / 2, j}^{n+1 / 2}=u_{i+1 / 2, j}^{n+1 / 2}-\frac{\phi_{i+1, j}-\phi_{i j}}{h}, \quad \tilde{v}_{i, j+1 / 2}^{n+1 / 2}=v_{i, j+1 / 2}^{n+1 / 2}-\frac{\phi_{i, j+1}-\phi_{i j}}{h} .
$$

The convective terms are discretized as

$\left(\mathbf{u} \cdot \nabla_{d} \mathbf{u}\right)_{i j}^{n+1 / 2}$

$$
=\frac{\tilde{u}_{i+1 / 2, j}^{n+1 / 2}+\tilde{u}_{i-1 / 2, j}^{n+1 / 2}}{2 h}\left(\overline{\mathbf{u}}_{i+1 / 2, j}^{n+1 / 2}-\overline{\mathbf{u}}_{i-1 / 2, j}^{n+1 / 2}\right)+\frac{\tilde{v}_{i, j+1 / 2}^{n+1 / 2}+\tilde{v}_{i, j-1 / 2}^{n+1 / 2}}{2 h}\left(\overline{\mathbf{u}}_{i, j+1 / 2}^{n+1 / 2}-\overline{\mathbf{u}}_{i, j-1 / 2}^{n+1 / 2}\right),
$$

$\left(\mathbf{u} \cdot \nabla_{d} \mathbf{c}\right)_{i j}^{n+1 / 2}$

$$
=\frac{\tilde{u}_{i+1 / 2, j}^{n+1 / 2}+\tilde{u}_{i-1 / 2, j}^{n+1 / 2}}{2 h}\left(\overline{\mathbf{c}}_{i+1 / 2, j}^{n+1 / 2}-\overline{\mathbf{c}}_{i-1 / 2, j}^{n+1 / 2}\right)+\frac{\tilde{v}_{i, j+1 / 2}^{n+1 / 2}+\tilde{v}_{i, j-1 / 2}^{n+1 / 2}}{2 h}\left(\overline{\mathbf{c}}_{i, j+1 / 2}^{n+1 / 2}-\overline{\mathbf{c}}_{i, j-1 / 2}^{n+1 / 2}\right),
$$

where the edge values $\overline{\mathbf{c}}_{i \pm 1 / 2, j}^{n+1 / 2}, \quad \overline{\mathbf{u}}_{i \pm 1 / 2, j}^{n+1 / 2}, \quad \overline{\mathbf{c}}_{i, j \pm 1 / 2}^{n+1 / 2}$, and $\overline{\mathbf{u}}_{i, j \pm 1 / 2}^{n+1 / 2}$ are computed using projected velocity fields, $\tilde{u}, \tilde{v}$, and a fifth order WENO algorithm [26].

\subsection{Discretization of surface tension terms}

In this section we describe the finite difference approximation to the surface tension term, $\mathbf{F}_{\mathrm{st}}$. Let

$$
\begin{aligned}
\mathbf{F}_{\mathrm{st}} & =-\sum_{k=1}^{3} \frac{\epsilon \beta}{\mathrm{We}_{k}}\left(f_{k}, g_{k}\right)=\sum_{k=1}^{3} \frac{\epsilon \beta}{\mathrm{We}_{k}} \nabla \cdot\left(\left|\nabla c_{k}\right|^{2} I-\nabla c_{k} \otimes \nabla c_{k}\right) \\
& =-\sum_{k=1}^{3} \frac{\epsilon \beta}{\mathrm{We}_{k}}\left(\partial_{x} c_{k} \partial_{y y} c_{k}-\partial_{y} c_{k} \partial_{x y} c_{k}, \partial_{y} c_{k} \partial_{x x} c_{k}-\partial_{x} c_{k} \partial_{x y} c_{k}\right) .
\end{aligned}
$$

Then the surface tension force components $\left(f_{1}, g_{1}\right)$ are discretized as

$$
\begin{aligned}
\left(f_{1}\right)_{i j}= & \frac{1}{2 h^{3}}\left(c_{1, i+1, j}-c_{1, i-1, j}\right)\left(c_{1, i, j+1}-2 c_{1 i j}+c_{1, i, j-1}\right) \\
& -\frac{1}{8 h^{3}}\left(c_{1, i, j+1}-c_{1, i, j-1}\right)\left(c_{1, i+1, j+1}+c_{1, i-1, j-1}-c_{1, i+1, j-1}-c_{1, i-1, j+1}\right), \\
\left(g_{1}\right)_{i j}= & \frac{1}{2 h^{3}}\left(c_{1, i, j+1}-c_{1, i, j-1}\right)\left(c_{1, i+1, j}-2 c_{1 i j}+c_{1, i-1, j}\right) \\
& -\frac{1}{8 h^{3}}\left(c_{1, i+1, j}-c_{1, i-1, j}\right)\left(c_{1, i+1, j+1}+c_{1, i-1, j-1}-c_{1, i+1, j-1}-c_{1, i-1, j+1}\right)
\end{aligned}
$$


and the other components, $\left(f_{2}, g_{2}\right)$ and $\left(f_{3}, g_{3}\right)$, are similarly defined by replacing $c_{1}$ in $(3.5)$ and (3.6) by $c_{2}$ and $1-c_{1}-c_{2}$, respectively. Further, $\mathbf{F}_{\mathrm{st}}^{n+1 / 2}$ in 3.3 is evaluated using $\mathbf{c}^{n+1 / 2}=$ $\left(\mathbf{c}^{n}+\mathbf{c}^{n+1}\right) / 2$.

\subsection{The discrete energy functional}

The discrete energy functional for the Boussinesq approximation is

$$
E_{h}(t)=\frac{1}{2}(\mathbf{u}, \mathbf{u})_{h}+\sum_{k=1}^{3} \frac{1}{M_{k} \mathrm{We}_{k}}\left(\left(F_{k}, 1\right)_{h}+\frac{\epsilon_{k}^{2}}{4}\left|c_{k}\right|_{1}^{2}\right)
$$

where $(f, g)_{h}=h^{2} \sum_{i=1}^{N_{x}} \sum_{j=1}^{N_{y}} f_{i j} g_{i j}$ and $|g|_{1}^{2}=\sum_{i=1}^{N_{x}} \sum_{j=1}^{N_{y}}\left(\left(g_{i+1, j}-g_{i j}\right)^{2}+\left(g_{i, j+1}-g_{i j}\right)^{2}\right)$ taking into account the Neumann boundary conditions to handle the boundary terms. Following the analysis in [29], it can be shown that if the time step $\Delta t$ is small enough, the discrete energy $E_{h}$ is nonincreasing. In practice, we find that $E_{h}$ is nonincreasing for $\Delta t$ such that our multigrid methods converge.

We note that an alternate discretization may be developed following our work on phase-field models of two-phase flows [30]. In that work, we formulated a version of the projection method using a special discretization of the Navier-Stokes equations such that the coupled discrete NavierStokes-Cahn-Hilliard system had a nonincreasing discrete energy functional for any value of $\Delta t$. We find that the scheme presented here results in slightly better accuracy than that used in [30].

\section{Numerical experiments}

In this section, we demonstrate convergence of our scheme numerically and simulate a threephase contact angle, a buoyancy-driven compound drop, and liquid/liquid remediation enhanced by the Rayleigh-Taylor instability. Unless otherwise specified, we use the free energy function $F(\mathbf{c})$ defined by 2.53. From our choice of the homogeneous free energy density 2.53 and an equilibrium profile 2.51, the concentration field varies from 0.05 to 0.95 over a distance of about $4 \sqrt{2} \epsilon \tanh ^{-1}(0.9)$. We have 4-8 grid points across interface in most of our simulations. Note that since we are taking a polynomial form of the free energy, $F(\mathbf{c})$, we may have nonphysical value of concentration (i.e. less than zero and greater than one), but its deviations from the physical values are negligible (in most of the cases, the deviations are of order $10^{-3}$ ). The time step is determined by restrictions due to the CFL condition, gravity, viscosity, and surface tension [42]:

$$
\Delta t<\min _{\Omega}\left(\min _{k=1,2,3} \sqrt{\frac{\left(\rho_{1}+\rho_{2}+\rho_{3}\right) \mathrm{We}_{k} \mathrm{We}_{\mathrm{rem}(k, 3)+1}}{8 \pi\left(\mathrm{We}_{k}+\mathrm{We}_{\mathrm{rem}(k, 3)+1}\right)}} h^{3 / 2}, \frac{3 \operatorname{Re} \rho^{n} h^{2}}{14 \eta^{n}}, \frac{h}{\left|\mathbf{u}^{n}\right|}, \sqrt{\frac{2 h}{\left|\mathcal{F}^{n}\right|}}\right),
$$

where $\mathcal{F}^{n}$ is the right hand side of 2.55 at time $t=n \Delta t$.

\subsection{Convergence test}

To obtain an estimate of the rate of convergence, we perform a number of simulations for a sample initial problem on a set of increasingly finer grids. The initial data is

$$
c_{1}(x, y, 0)=\frac{1}{2}\left(1-\tanh \left(\frac{y-1 / 3}{2 \sqrt{2} \epsilon}\right)\right),
$$




$$
c_{2}(x, y, 0)=\frac{1}{2}\left(\tanh \left(\frac{y-1 / 3}{2 \sqrt{2} \epsilon}\right)-\tanh \left(\frac{y-2 / 3}{2 \sqrt{2} \epsilon}\right)\right) .
$$

That is, narrow transition layers separate three immiscible fluids. The initial velocity is a swirling flow

$$
(u(x, y, 0), v(x, y, 0))=\left(-0.25 \sin ^{2}(\pi x) \sin (2 \pi y), 0.25 \sin ^{2}(\pi y) \sin (2 \pi x)\right)
$$

on a domain $\Omega=(0,1) \times(0,1)$. No-slip boundary conditions are applied to top and bottom planes, and periodic ones to the side walls. The numerical solutions are computed on the uniform grids, $h=1 / 2^{n}$ for $n=5,6,7,8$, and 9 . For each case, the calculations are run to time $T=0.2$, the uniform time steps, $\Delta t=0.1 h, \mathrm{Re}=10, \mathrm{We}_{1}=\mathrm{We}_{2}=\mathrm{We}_{3}=100, \mathrm{Pe}=2$, and $\epsilon=0.005 \sqrt{2}$, are used to establish the convergence rates. Figs. 4.1 (a) and (b) show the initial configuration and a snapshot of the solution at time $T=0$ and $T=0.2$, respectively, with $h=1 / 64$ and $\operatorname{Re}=100$.

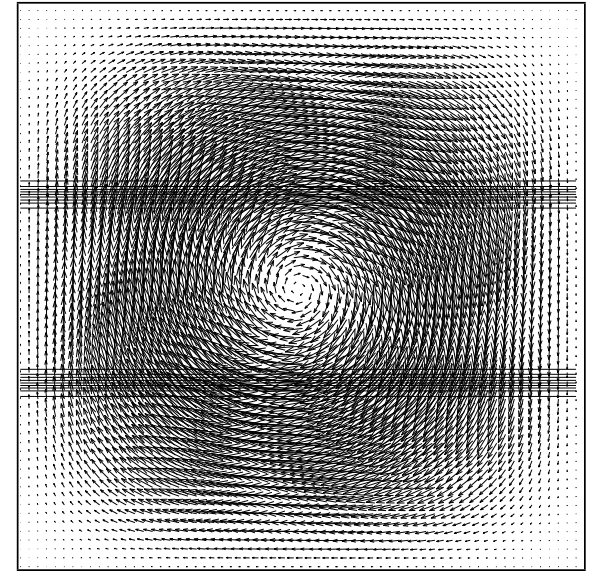

(a)

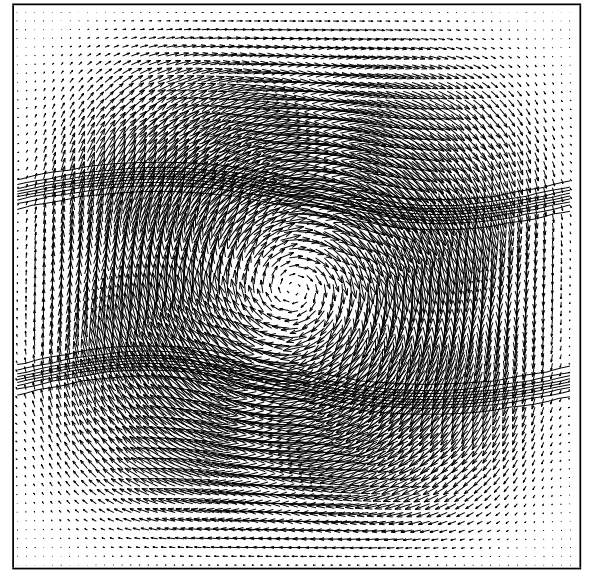

(b)

FIG. 4.1. Snapshots of the solutions at (a) $T=0$ and (b) $T=0.2$. The uppermost layer is fluid 3, the middle layer is fluid 2, and the lower layer is fluid 1 . The contour lines are from $c_{2}=0.1$ to $c_{2}=0.9$ with 0.1 step size.

Since a cell centered grid is used, we define the error to be the difference between that grid and the average of the next finer grid cells covering it:

$$
e_{h / \frac{h}{2}}:=c_{h i j}-\left(c_{\frac{h}{2}}{ }_{2 i, 2 j}+c_{\frac{h}{2} 2 i-1,2 j}+c_{\frac{h}{2} 2 i, 2 j-1}+c_{\frac{h}{2} 2 i-1,2 j-1}\right) / 4
$$

TABLE 4.1

Convergence results with $\operatorname{Re}=10: u, v, c_{1}$, and $c_{2}$

\begin{tabular}{cccccccc}
\hline Case & $32-64$ & $64-128$ & rate & $128-256$ & rate & $256-512$ & rate \\
\hline$u$ & $5.4530 \mathrm{e}-4$ & $4.4986 \mathrm{e}-5$ & 3.5995 & $1.0895 \mathrm{e}-5$ & 2.0458 & $2.7798 \mathrm{e}-6$ & 1.9706 \\
$v$ & $6.8462 \mathrm{e}-4$ & $2.9858 \mathrm{e}-5$ & 4.5191 & $7.5194 \mathrm{e}-6$ & 1.9894 & $1.8850 \mathrm{e}-6$ & 1.9961 \\
$c_{1}$ & $2.9211 \mathrm{e}-2$ & $3.1969 \mathrm{e}-3$ & 3.1918 & $7.5863 \mathrm{e}-4$ & 2.0752 & $1.8755 \mathrm{e}-4$ & 2.0161 \\
$c_{2}$ & $4.1640 \mathrm{e}-2$ & $4.2759 \mathrm{e}-3$ & 3.2836 & $1.0037 \mathrm{e}-3$ & 2.0908 & $2.4801 \mathrm{e}-4$ & 2.0169 \\
\hline
\end{tabular}


TABLE 4.2

Convergence results with $\mathrm{Re}=100: u, v, c_{1}$, and $c_{2}$

\begin{tabular}{cccccccc}
\hline Case & $32-64$ & $64-128$ & rate & $128-256$ & rate & $256-512$ & rate \\
\hline$u$ & $7.9061 \mathrm{e}-4$ & $2.1666 \mathrm{e}-4$ & 1.8675 & $6.3568 \mathrm{e}-5$ & 1.7691 & $1.8657 \mathrm{e}-5$ & 1.7686 \\
$v$ & $1.8242 \mathrm{e}-4$ & $4.4618 \mathrm{e}-5$ & 2.0316 & $1.4104 \mathrm{e}-5$ & 1.6615 & $4.4661 \mathrm{e}-6$ & 1.6590 \\
$c_{1}$ & $1.6055 \mathrm{e}-2$ & $3.1327 \mathrm{e}-3$ & 2.3576 & $7.6571 \mathrm{e}-4$ & 2.0325 & $1.9041 \mathrm{e}-4$ & 2.0077 \\
$c_{2}$ & $2.5432 \mathrm{e}-2$ & $4.4223 \mathrm{e}-3$ & 2.5238 & $1.0388 \mathrm{e}-3$ & 2.0899 & $2.5764 \mathrm{e}-4$ & 2.0114 \\
\hline
\end{tabular}

The rate of convergence is defined as the ratio of successive errors in the discrete $l_{2}$-norm:

$$
\log _{2}\left(\left\|e_{h / \frac{h}{2}}\right\| /\left\|e_{\frac{h}{2} / \frac{h}{4}}\right\|\right)
$$

The errors and rates of convergence with $\mathrm{Re}=10$ and $\mathrm{Re}=100$ are given in Tables 4.1 and 4.2, respectively. The results suggest that the scheme is indeed second order accurate when the $\mathrm{Re}$ number is small. When $\operatorname{Re}=100$, the rate of convergence decreases.

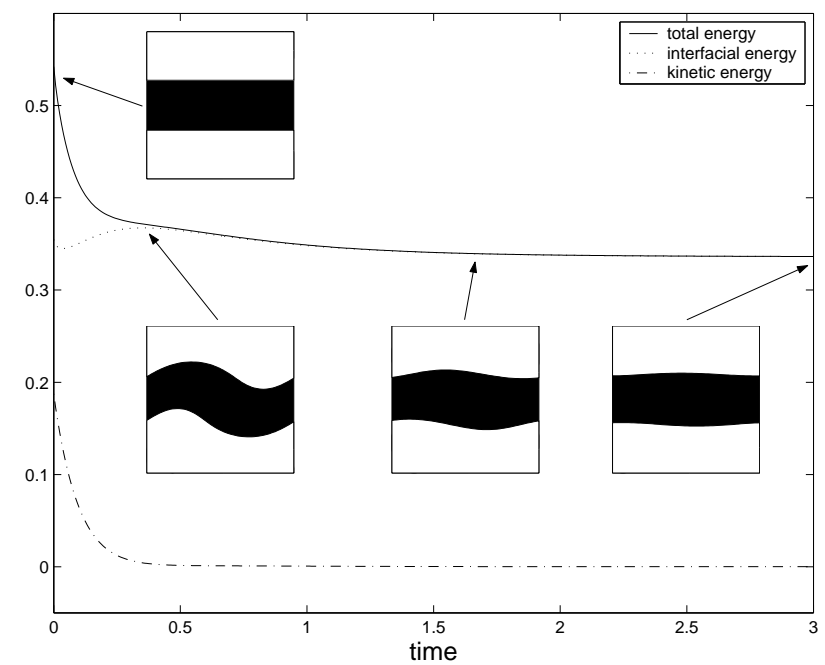

FIG. 4.2. The time dependent energies of the numerical solutions with the initial data 4.1 -4.6 and with $\operatorname{Re}=10$ and $\mathrm{We}_{1}=\mathrm{We}_{2}=\mathrm{We}_{3}=10$. Snapshots of the concentration field $c_{2}$ are shown with filled contour at $c_{2}=0.5$.

We also examined the time evolution of the total energy (with $F$ and $F_{k}$ defined as in (3.7) and 2.53 respectively). The results are shown in Fig. 4.2 together with the interfacial (dotted) and kinetic energy (dot-dashed) components. The interfacial energy contains both the gradient and Helmholtz free energy terms. The solid curve marks the total energy. The insets show the layer morphology ( $c_{2}=0.5$ filled contour) at various times. The initial data, boundary conditions and all the parameters are the same as considered previously except that the Weber numbers are $\mathrm{We}_{1}=\mathrm{We}_{2}=\mathrm{We}_{3}=10$ so that surface tension plays a more important role in the evolution. 
Here the Reynolds number $\mathrm{Re}=10$. Observe that the total energy is nonincreasing throughout the evolution. The kinetic energy also monotonically decreases due to viscous dissipation. The interfacial energy is nonmonotone, however. At early times, the initially flat layer deforms and the surface energy increases. Around approximately $t=0.4$, the surface energy reaches a peak due to the increased deformation of the layer and then decreases towards an equilibrium value corresponding to a flat layer (two interfaces). When the layer is most deformed, the surface tension force induces a reversal of the velocity field such that the deformed layer relaxes back to a flat configuration at later times.

\subsection{Contact angles}

Following [40], we next investigate the spreading of a liquid lens consisting of an initially circular immiscible droplet of fluid located at an interface between two other immiscible fluids. See Fig. 4.3.a).

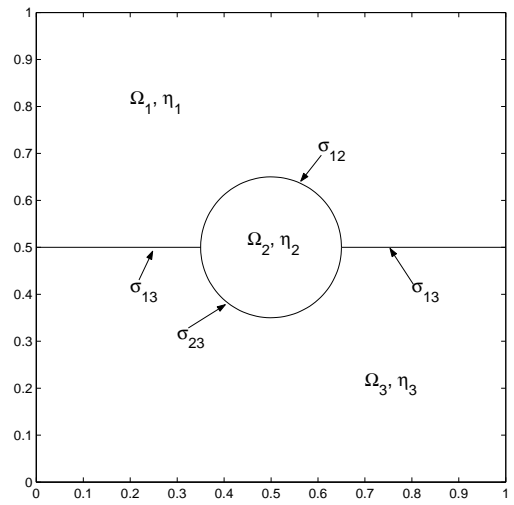

(a)

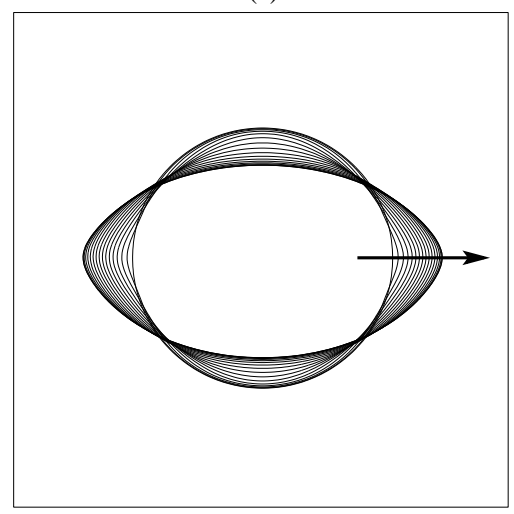

(c)

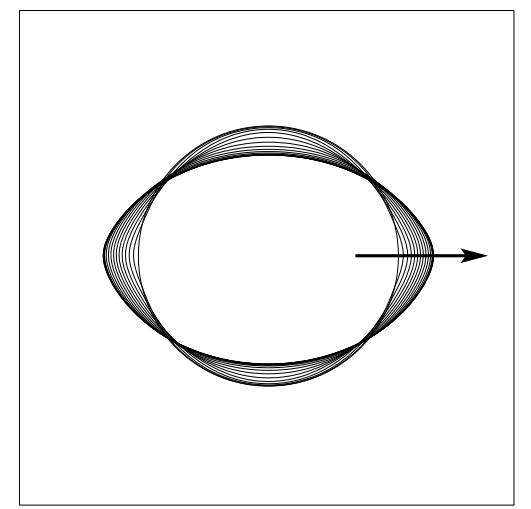

(b)

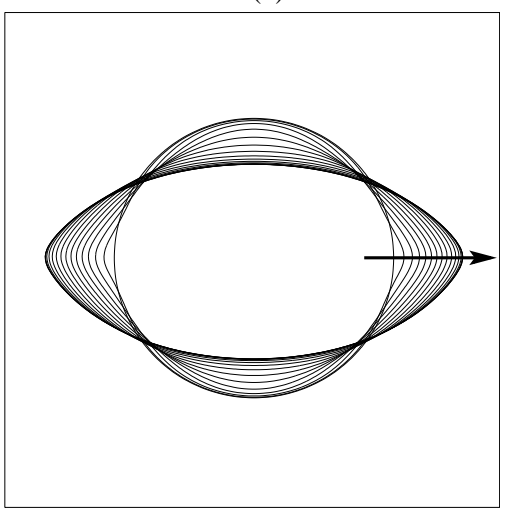

(d)

FIG. 4.3. (a) Initial configuration: the upper fluid is phase 1, the lower fluid is phase 3, and the droplet is phase 2. (b), (c), and (d) are evolutions of initial circular drop for $\mathrm{We}_{2}=60$ and $\mathrm{We}_{1}=\mathrm{We}_{3}=108,60,36$, respectively. The arrow shows the direction of the evolution, the most deformed lines in (b), (c), and (d) are corresponding steady shapes and the enclosing box size is $[0.23,0.77] \times[0.23,0.77]$. 
The initial condition is a circular droplet, $\Omega_{2}$ (located at a free surface between $\Omega_{1}$ and $\Omega_{3}$ ) and the initial velocity is zero, i.e.,

$$
\begin{aligned}
& c_{1}(x, y, 0)=\max \left[0.5\left(1+\tanh \left(\frac{y-0.5}{2 \sqrt{2} \epsilon}\right)\right)-c_{2}(x, y), 0\right], \\
& c_{2}(x, y, 0)=0.5\left(1+\tanh \left(\frac{0.15-\sqrt{(x-0.5)^{2}+(y-0.5)^{2}}}{2 \sqrt{2} \epsilon}\right)\right), \\
& u(x, y, 0)=v(x, y, 0)=0 .
\end{aligned}
$$

The computational domain is $\Omega=[0,1] \times[0,1]$. The fluids are density and viscosity matched $(\rho=1, \eta=1)$ and

$$
\mathrm{Re}=60, \quad \mathrm{We}_{2}=60, \quad \mathrm{We}_{1}=\mathrm{We}_{3}=108,60,36 .
$$

In Figs. 4.3 b) $-(\mathrm{d})$, the evolution of the $c_{2}=1 / 2$ contour line is shown for three cases with $\mathrm{Re}=60$ and $\mathrm{We}_{2}=60, \mathrm{We}_{1}=\mathrm{We}_{3}=108,60,36$, respectively. In all cases, $\epsilon=0.005 \sqrt{2}$, $\mathrm{Pe}=100 / \epsilon, h=1 / 256$, and $\Delta t=0.25 h$. As the droplet spreads, it reaches an equilibrium shape. The most deformed curve in each figure is the numerical steady state. Theoretically, the shape of the steady state drop is controlled by the drop volume and the three surface tensions (inverse Weber numbers). The equilibrium three-phase contact angle is determined by

$$
\frac{\sin \theta_{1}}{\frac{1}{\mathrm{We}_{2}}+\frac{1}{\mathrm{We}_{3}}}=\frac{\sin \theta_{2}}{\frac{1}{\mathrm{We}_{1}}+\frac{1}{\mathrm{We}_{3}}}=\frac{\sin \theta_{3}}{\frac{1}{\mathrm{We}_{1}}+\frac{1}{\mathrm{We}_{2}}}
$$

and the relation between the lens area $A$, its length $d$ (the distance between triple junctions), and the contact angles $\theta_{i}$ of the $i$ th phase (Young's law) is

$$
d=\left(\frac{1}{8 A}\left(\frac{2\left(\pi-\theta_{1}\right)-\sin \left(2\left(\pi-\theta_{1}\right)\right)}{\sin ^{2}\left(\pi-\theta_{1}\right)}+\frac{2\left(\pi-\theta_{3}\right)-\sin \left(2\left(\pi-\theta_{3}\right)\right)}{\sin ^{2}\left(\pi-\theta_{3}\right)}\right)\right)^{-1 / 2} .
$$

Thus, the accuracy of the steady lens shape can be measured by comparing the observed $d$ with the analytical value.

The evolution of $d$ for the three cases is shown in Fig. 4.4. The numerical value of $d$ is obtained from the $c_{2}=1 / 2$ contour line. Note that in all cases, there is rapid increase in $d$ at early times followed by a slow approach to equilibrium. Note also there is an overshoot in the early evolution of the $\mathrm{We}_{3}=36$ and $\mathrm{We}_{3}=108$ cases. In Table 4.3, the equilibrium values of $d$ are shown for the three cases together with the corresponding theoretical values. There is very good agreement between the theory and simulation.

TABLE 4.3

Equilibrium measurements

\begin{tabular}{cccc}
\hline $\mathrm{We}_{1}, \mathrm{We}_{2}, \mathrm{We}_{3}$ & $d_{\text {exact }}$ & $d_{\text {numerical }}$ & $d_{\text {numerical }}, \epsilon / 2$ \\
\hline $108,60,108$ & 0.3746 & 0.3982 & $\cdot$ \\
$60,60,60$ & 0.4138 & 0.4368 & $\cdot$ \\
$36,60,36$ & 0.4578 & 0.4622 & 0.4502 \\
\hline
\end{tabular}




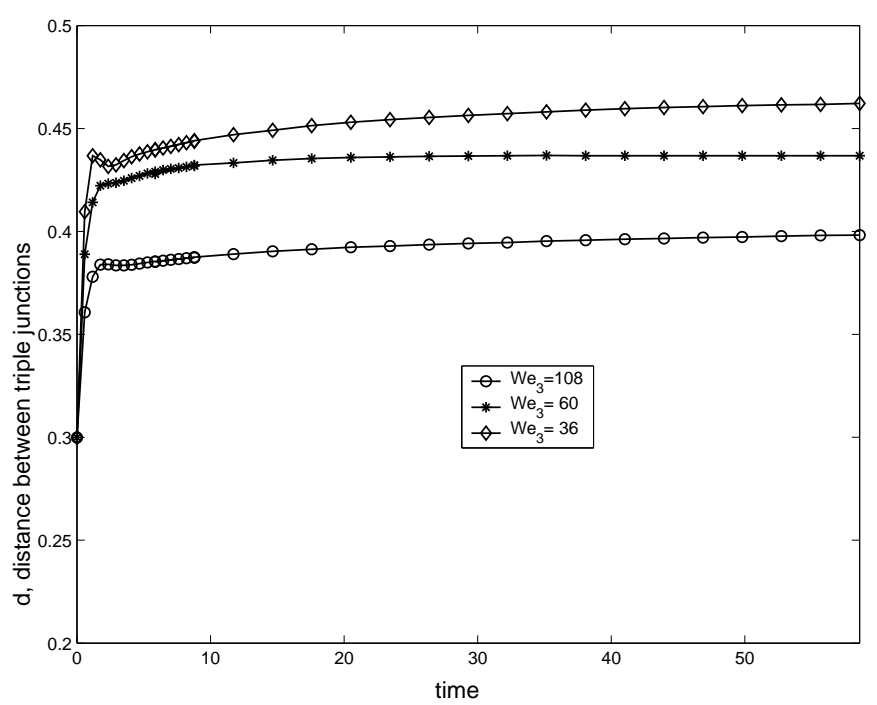

FIG. 4.4. Time evolution of $d$, distance between triple junctions. $\mathrm{We}_{3}=108(\circ), \mathrm{We}_{3}=60(*)$, and $\mathrm{We}_{3}=36(\diamond)$.

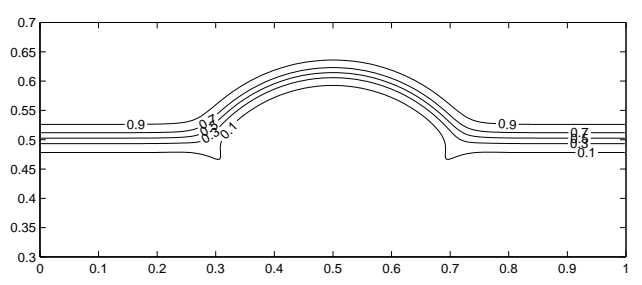

(a)

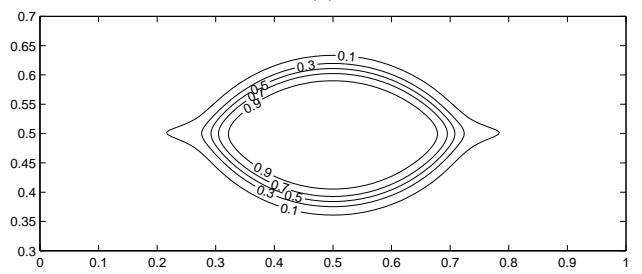

(a)

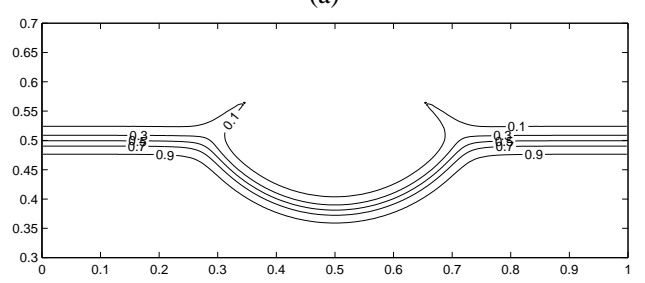

(a)

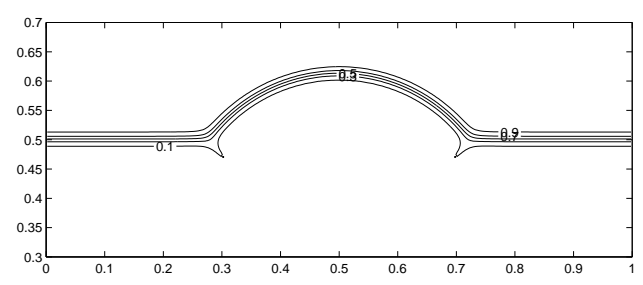

(b)

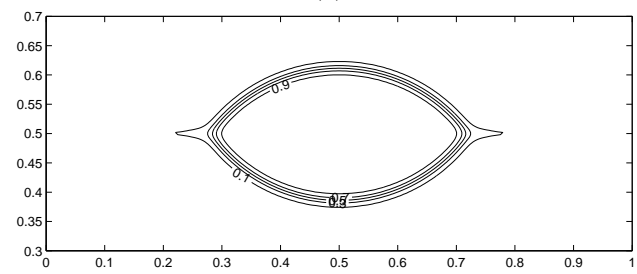

(b)

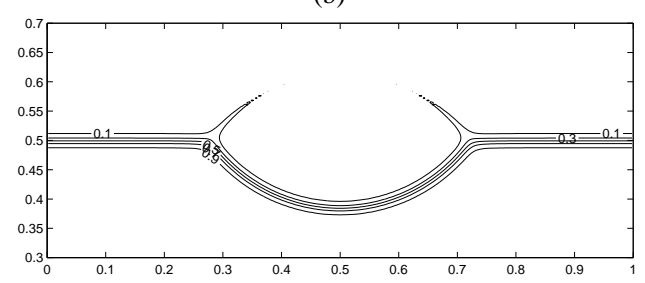

(b)

FIG. 4.5. Contour lines of concentration $c_{2}$ at the five levels $c_{2}=0.1,0.3,0.5,0.7$, and 0.9 in the case of $\mathrm{We}_{1}=\mathrm{We}_{3}=36$ and $\mathrm{We}_{2}=60$. Left column: $\epsilon=0.005 \sqrt{2}$ and right column: $\epsilon=0.0025 \sqrt{2}$, respectively. 
In Fig. 4.5. a) and (b), we plot the contours of the concentration $c_{2}$ at the five levels $c_{2}=$ $0.1,0.3,0.5,0.7$, and 0.9 for the case with $\mathrm{We}_{1}=\mathrm{We}_{3}=36$ and $\mathrm{We}_{2}=60$. In the left column $\epsilon=0.005 \sqrt{2}$ while in the right column $\epsilon=0.0025 \sqrt{2}$. Not only do the concentration contours appear to converge, also note that when $\epsilon=0.0025 \sqrt{2}$, we obtain $d_{\text {numerical }}=0.4502$, which is closer to the exact result than is the case with $\epsilon=0.005 \sqrt{2}$. Interestingly, the $c_{2}=0.1$ contour appears to be elongated near the triple junctions-a fact that seems to persist under refinement of $\epsilon$.

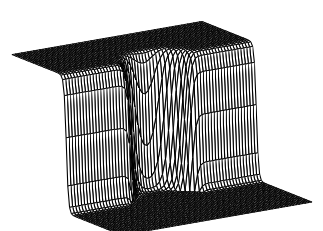

(a1)

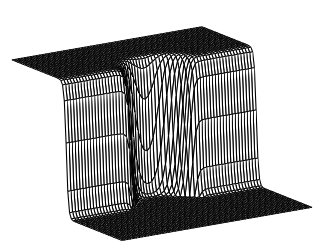

(b1)

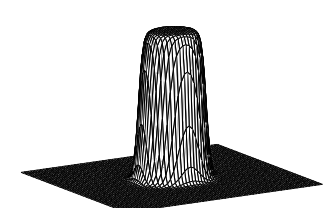

(a2)

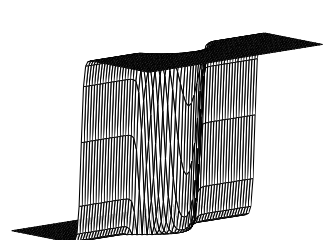

(b2)

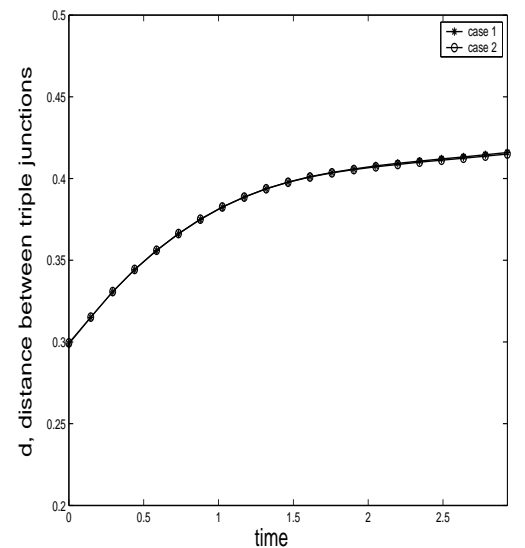

(c)

FIG. 4.6. Initial concentration profiles; case 1: (a1) $c_{1}$ and (a2) $c_{2}$, case 2: (b1) $c_{1}$ and (b2) $c_{2}$. Time evolution of $d$, distance between triple junctions for the two cases (case $1(*)$ and case $2(0)$ ) with $\mathrm{We}_{3}=60$.

We next show that numerical results are not sensitive to the choice of component labels. We take the same initial configuration but change the component labels, i.e.

$$
\begin{aligned}
& c_{1}(x, y, 0)=\max \left[0.5\left(1+\tanh \left(\frac{y-0.5}{2 \sqrt{2} \epsilon}\right)\right)-c_{3}(x, y), 0\right], \\
& c_{3}(x, y, 0)=0.5\left(1+\tanh \left(\frac{0.15-\sqrt{(x-0.5)^{2}+(y-0.5)^{2}}}{2 \sqrt{2} \epsilon}\right)\right) .
\end{aligned}
$$

We then solve the equations for the concentrations, $c_{1}$ and $c_{2}=1-c_{1}-c_{3}$ with $\mathrm{We}_{1}=\mathrm{We}_{2}=$ $\mathrm{We}_{3}=60$. In Fig. 4.6, the initial concentration profiles are shown: for the original case (hereafter referred to as case 1) (a1) $c_{1}$ and (a2) $c_{2}$, and for the new case (referred to as case 2) (b1) $c_{1}$ and (b2) $c_{2}$. The time evolution of the distance $d$ between the triple junctions for the two cases (case 1 $(*)$ and case $2(0)$ ) is shown in (c). As can be seen from this figure, the values of $d$ from these two cases are essentially identical and thus the results are insensitive to label switching.

In Fig. 4.7 the time evolution of total energy (with $F$ and $F_{k}$ defined as in (3.7) and 2.53 ) respectively) is shown together with the corresponding interfacial and kinetic energy components of the numerical solutions. In addition, the filled contour $c_{2}=0.5$ is shown at various times indicating the corresponding morphology of the drop. The initial data are (4.4)- 4.6 and the parameters correspond to the case in which $\mathrm{We}_{1}=\mathrm{We}_{3}=36$ and $\mathrm{We}_{2}=60$. In the figure, the solid line is the total energy, the dotted line is interfacial energy, and the dash-dotted line is the kinetic energy. The inset shows a blow-up of the kinetic energy. Since the initial velocity is zero and the evolution 


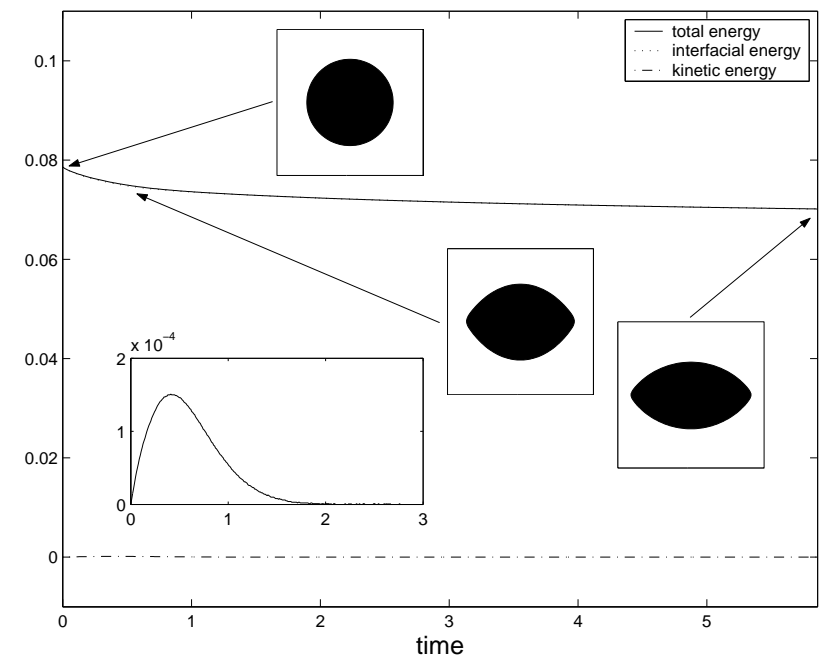

FIG. 4.7. The time dependent energies of the numerical solutions with the initial data $4.4-\sqrt{4.6}$ and the case with We $1=$ $\mathrm{We}_{3}=36$ and $\mathrm{We}_{2}=60$. Snapshots of the concentration field $c_{2}$ are shown with filled contour at $c_{2}=0.5$.

is primarily surface energy driven, the kinetic energy is very small. At early times, a small amount of surface energy is transferred to kinetic energy as the drop begins to elongate. At later times, the kinetic energy rapidly decays as the drop approaches its equilibrium configuration. As expected, the total energy monotonically decreases to its equilibrium value. Correspondingly, the interfacial energy also decreases.

Next, we consider a similar problem in three dimensions. We place a periodic array of spheres on an interface between two immiscible fluids analogously to that shown in Fig. 4.3 a) in two dimensions. The computational domain is $\Omega=[0,1] \times[0,1] \times[0,1]$ and the mesh size is $64 \times$ $64 \times 64$ with time step $\Delta t=0.001, \epsilon=0.008 \sqrt{2}$, and $\mathrm{Pe}=100 / \epsilon$. No-slip boundary conditions for the top and bottom planes and periodic boundary conditions for the side walls are applied. Specifically, the initial data in a single period box are

$$
\begin{aligned}
& c_{1}(x, y, z)=\max \left[0.5\left(1+\tanh \left(\frac{z-0.5}{2 \sqrt{2} \epsilon}\right)\right)-c_{2}(x, y), 0\right], \\
& c_{2}(x, y, z)=0.5\left(1+\tanh \left(\frac{0.35-\sqrt{(x-0.5)^{2}+(y-0.5)^{2}+(z-0.5)^{2}}}{2 \sqrt{2} \epsilon}\right)\right), \\
& u(x, y, z)=v(x, y, z)=w(x, y, z)=0 .
\end{aligned}
$$

We take the viscosities and densities of the components to be matched $\left(\eta_{1}=\eta_{2}=\eta_{3}=1\right)$ and $\left(\rho_{1}=\rho_{2}=\rho_{3}=1\right)$ with the following parameters:

$$
\mathrm{Re}=60, \quad \mathrm{We}_{1}=\mathrm{We}_{3}=36, \quad \mathrm{We}_{2}=60 .
$$

In Fig. 4.8. evolution is shown and only the spheres are visualized with a reference plane passing through the equators of the spheres. The actual interface deforms. As the drops spread out and flatten due to surface tension forces, the drops interact with their periodic neighbors. In this case, 


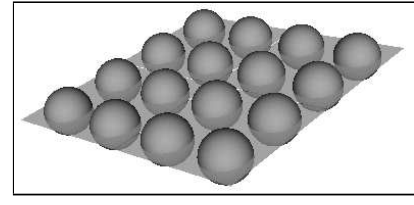

$\mathrm{t}=0.0$

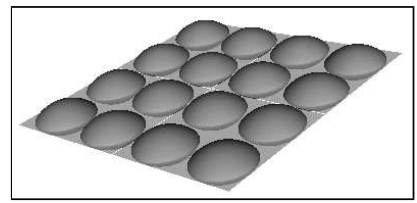

$\mathrm{t}=1.8$

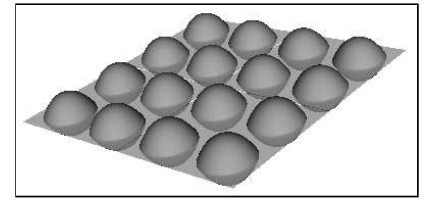

$\mathrm{t}=0.2$

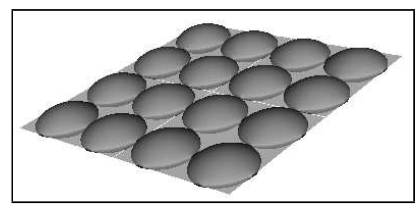

$\mathrm{t}=2.6$

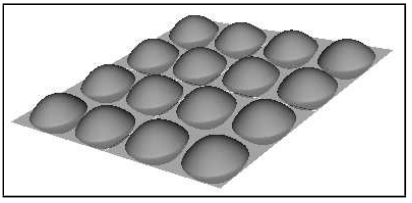

$\mathrm{t}=0.6$

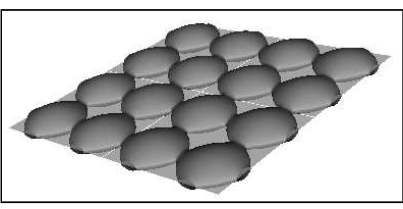

$\mathrm{t}=3.2$

FIG. 4.8. Evolution of spheres under surface tension forces; the nondimensional times are shown below each figure.

the distance between the neighbors is less than the equilibrium length of an isolated drop. As seen in Fig. 4.8, this leads to merger with the periodic images and results in a lattice-like microstructure of the second fluid on the interface between the two other immiscible fluids.

\subsection{Numerical simulation of a buoyancy-driven compound drop}

In this section, a buoyancy-driven evolution of a 3D compound drop is investigated. In Fig. 4.9, a schematic diagram of the initial configuration is shown. The three fluids are immiscible where a heavy droplet of fluid I is encapsulated by a light fluid II. Fluid I is the heaviest component. This models a flow in which a heavy fluid contains a dispersed contaminant. Releasing drops of the light fluid II from the bottom of the container provides the means to encapsulate the contaminants. Restricting this to a single drop yields the initial condition we consider.

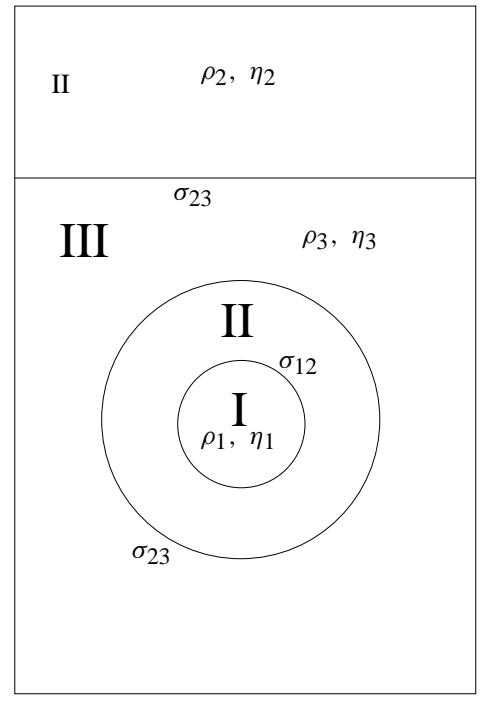

FIG. 4.9. Scheme of a compound drop. 
Specifically, the initial data are

$$
\begin{aligned}
& c_{1}(x, y, z)=0.5\left(1+\tanh \left(\frac{0.3-r}{2 \sqrt{2} \epsilon}\right)\right), \\
& c_{2}(x, y, z)=0.5\left(2+\tanh \left(\frac{0.5-r}{2 \sqrt{2} \epsilon}\right)+\tanh \left(\frac{z-2.5}{2 \sqrt{2} \epsilon}\right)\right)-c_{1}(x, y, z), \\
& u(x, y, z)=v(x, y, z)=w(x, y, z)=0,
\end{aligned}
$$

where $r=\sqrt{(x-1)^{2}+(y-1)^{2}+(z-1.5)^{2}}$. We take the viscosities and surface tensions of the components to be matched $\left(\eta_{1}=\eta_{2}=\eta_{3}=1\right)$ and $\left(\sigma_{1}=\sigma_{2}=\sigma_{3}=1\right)$ with the following parameters:

$$
\rho_{1}=1.044, \quad \rho_{2}=0.957, \quad \rho_{3}=1, \quad \operatorname{Re}=36, \quad \mathrm{We}_{1}=\mathrm{We}_{2}=\mathrm{We}_{3}=1127, \quad \mathrm{Fr}=1 .
$$

The computational domain is $\Omega=[0,2] \times[0,2] \times[0,4]$ and the mesh size is $32 \times 32 \times 64$ with time step $\Delta t=0.002, \epsilon=0.01 \sqrt{2}$, and Pe $=10 / \epsilon$. No-slip boundary conditions for the top and bottom planes and periodic boundary conditions for the side walls are applied.

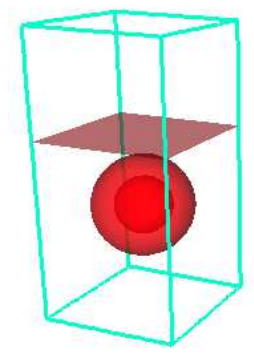

$\mathrm{t}=0.0$

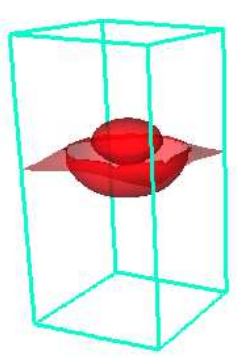

$\mathrm{t}=14.0$

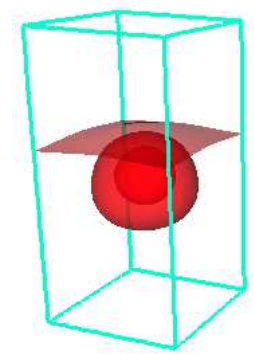

$\mathrm{t}=6.0$

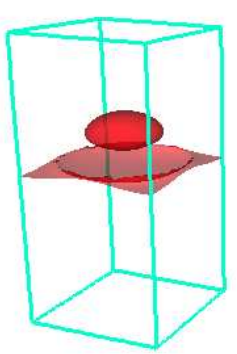

$\mathrm{t}=17.0$

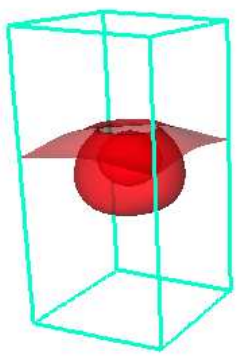

$\mathrm{t}=8.0$

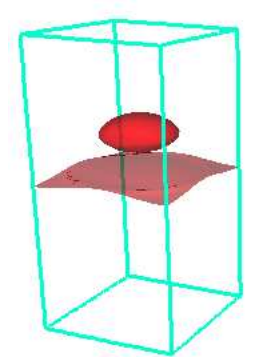

$\mathrm{t}=20.0$

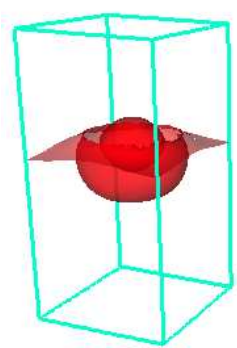

$\mathrm{t}=12.0$

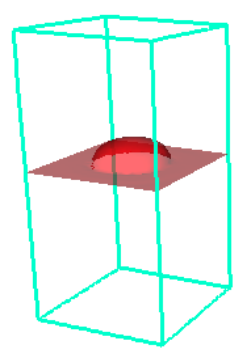

$\mathrm{t}=44.0$

FIG. 4.10. Evolution of a compound drop; the nondimensional times are shown below each figure.

The evolution is presented in Fig. 4.10. An upper (flat) interface separates the heavy ambient from same fluid that encapsulates the heavy drop. The compound drop is lighter than the heavy ambient and so it rises and deforms. The encapsulating fluid rises faster than the heavy inner drop but nevertheless the compound drop remains intact until it penetrates the upper interface. The heavy inner drop is carried upwards as the encapsulated fluid is released. The drop then falls back on the interface remaining trapped there by surface tension forces even though it is heavier than the 
lower ambient. At this point, the drop could be removed from the system by "sucking" it off the interface. Imagining that the heavy inner drop is a contaminant in the lower ambient, this provides a mechanism of liquid/liquid extraction by which fluid III may be cleansed.

\subsection{Rayleigh-Taylor instability of ternary fluid flows}

In this section, we exploit the fact that our ternary NSCH system is capable of describing multicomponent fluid flows containing immiscible, miscible and partially miscible components. The miscibility of the components is modeled through the properties of the free energy $F\left(c_{1}, c_{2}\right)$. It is nontrivial to construct free energies capable of describing partially miscible systems where, for example, two components are immiscible and the third component is preferentially miscible in one of the immiscible components. Nevertheless, we have been able to construct a class of such free energies, and one example is given below:

$$
F\left(c_{1}, c_{2}\right)=2 c_{1}^{2}\left(1-c_{1}-c_{2}\right)^{2}+\left(c_{1}+0.2\right)\left(c_{2}-0.2\right)^{2}+\left(1.2-c_{1}-c_{2}\right)\left(c_{2}-0.4\right)^{2} .
$$

A contour plot of the free energy $F\left(c_{1}, c_{2}\right)$ on the Gibbs triangle is shown in Fig. 4.11 The two minima of $F\left(c_{1}, c_{2}\right)$ are at $(0.7779,0.2330,-0.0109)$ and $(-0.0151,0.3651,0.6499)$. These minima lie very slightly outside the Gibbs triangle. As a demonstration of the evolution possible in partially miscible liquid systems, we present an example in which there is a gravity-driven (Rayleigh-Taylor) instability that enhances the transfer of a preferentially miscible contaminant from one immiscible fluid to another in 2D. The initial configuration is shown in Fig. 4.12 The top half of the domain consists of a mixture of fluid I and fluid II, and the bottom half consists of fluid III, which is immiscible with fluid I. Fluid II is preferentially miscible with fluid III. Fluid I is assumed to be the lightest and fluid II the heaviest. The density of the I/II mixture is heavier than that of fluid III, so the density gradient induces Rayleigh-Taylor instability.

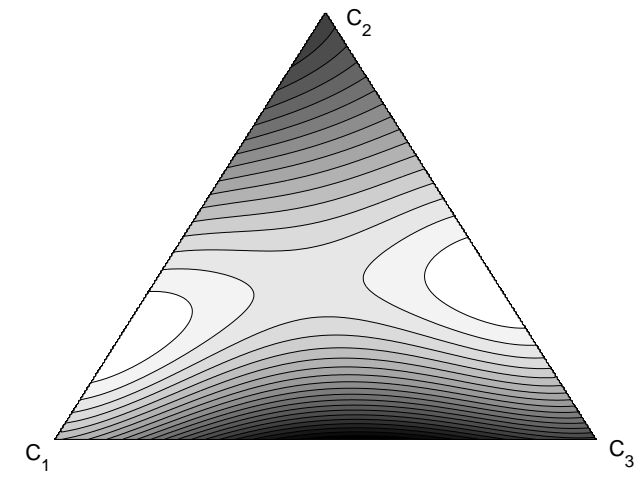

FIG. 4.11. Contour plot of the free energy $F\left(c_{1}, c_{2}\right)$ on the Gibbs triangle.

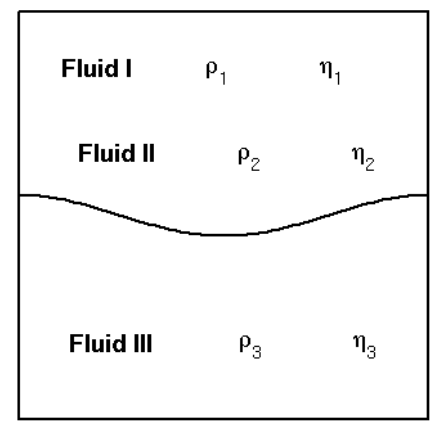

FIG. 4.12. Scheme of initial configuration

In particular, the initial data are

$$
\begin{aligned}
& c_{1}(x, y)=c_{2}(x, y)=0.25\left(1+\tanh \left(\frac{y-0.5-0.1 \cos (2 \pi x)}{2 \sqrt{2} \epsilon}\right)\right), \\
& u(x, y)=v(x, y)=0,
\end{aligned}
$$



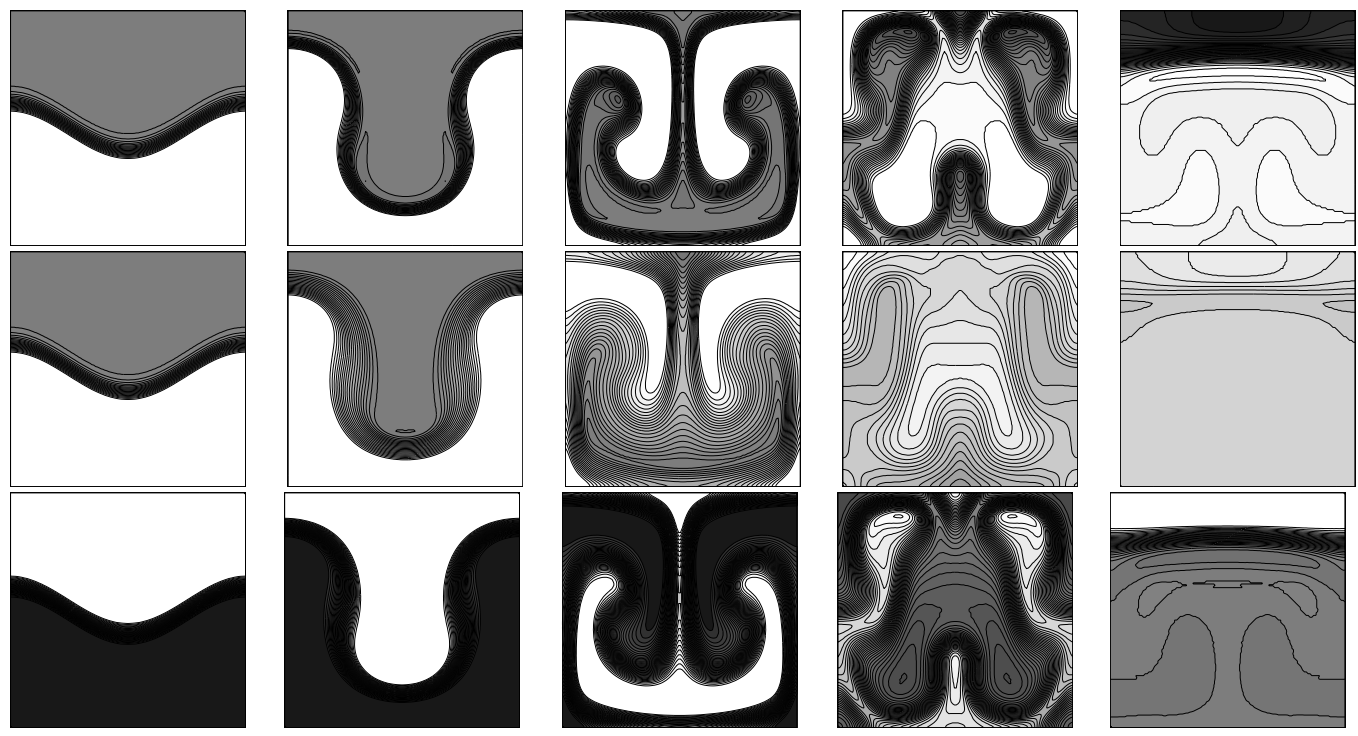

FIG. 4.13. Evolution of concentration of fluid I (top row), II (middle row), and III (bottom row). The contours of $c_{1}, c_{2}$, and $c_{3}$ are visualized in gray-scale where darker regions denote larger values of $c_{1}, c_{2}$, and $c_{3}$, respectively. Nondimensional times are $\mathrm{t}=0,3.91,7.81,15.63$, and 195.31.

and the simulation parameters are

$$
\rho_{1}=1, \rho_{2}=4, \rho_{3}=2, \mathrm{Fr}=1, \operatorname{Re}=313, \mathrm{We}_{1}=\mathrm{We}_{2}=\mathrm{We}_{3}=9.8 \times 10^{6} .
$$

The computational domain is $\Omega=[0,1] \times[0,1]$ and the mesh size is $128 \times 128$ with time step $\Delta t=0.5 h, \epsilon=0.01 \sqrt{2}$ and $\mathrm{Pe}=10 / \epsilon$. Here, we assume that the surface tension is small so that the interface undergoes the Rayleigh-Taylor instability (e.g. see paper [16] for the effect of surface tension on the instability).

The evolution of the three phases is shown in Fig. 4.13 The top row shows the evolution of fluid I, middle and bottom correspond to fluid II and fluid III, respectively. That is, the contours of $c_{1}, c_{2}$, and $c_{3}$ are visualized in gray-scale where darker regions denote larger values of $c_{1}, c_{2}$, and $c_{3}$, respectively. As the simulation begins, the I/II mixture falls and fluid II diffuses into fluid III. A characteristic Rayleigh-Taylor (inverted) mushroom forms, the surface area of the I/III interface increases, and vorticity is generated and shed into the bulk. As fluid II is diffused from fluid I, the pure fluid I rises to the top as shown in Fig. 4.13 Imagining that fluid II is a contaminant in fluid I, this configuration provides an efficient means of cleansing fluid I since the buoyancy-driven flow enhances the diffusional transfer of fluid II from fluid I to fluid III.

\subsection{Example of Adaptive Mesh Refinement-binary spinodal decomposition}

In the typical setting, solutions to the Cahn-Hilliard equation are nearly constant in the so-called "bulk" regions. Between the bulk regions, solutions exhibit thin transition layers, through which the solution takes on values intermediate to those in the bulk regions. Typically, the bulk regions comprise the largest portion, by far, of the computational domain. Since in most of the applications it is sufficient to finely resolve only the transition layers, fixed grid meshing represents a waste of 
computational resources. Thus, efficient, adaptive-mesh solvers for the $\mathrm{CH}$ equation, able to finely resolve only the diffuse interface, are highly desirable.

In preliminary work [32], we are currently adapting block-structured adaptive methods originally designed by Berger and Oliger [9] for conservation laws and general hyperbolic equations. The block-structured framework was adopted in the CHOMBO package [3], which we utilize. However, we do not use the multi-level multigrid algorithm from CHOMBO (see [3]), but rather we employ a nonlinear adaptive multigrid solver [44] following our work here and in [30, 31].

To illustrate the potential of this approach, we present preliminary results on adaptive mesh refinement for the binary $\mathrm{CH}$ equation from [32]. The governing equations are

$$
\begin{aligned}
\frac{\partial c}{\partial t} & =\Delta \mu, \\
\mu & =c^{3}-1.5 c^{2}+0.5 c-\epsilon^{2} \Delta c .
\end{aligned}
$$

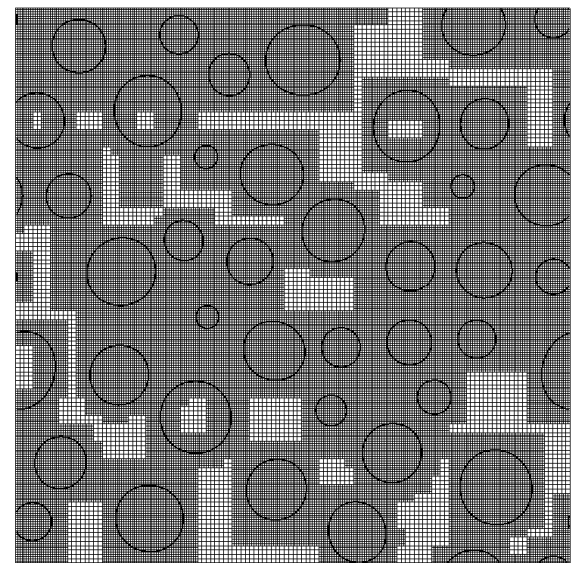

(a) $\mathrm{t}=2$

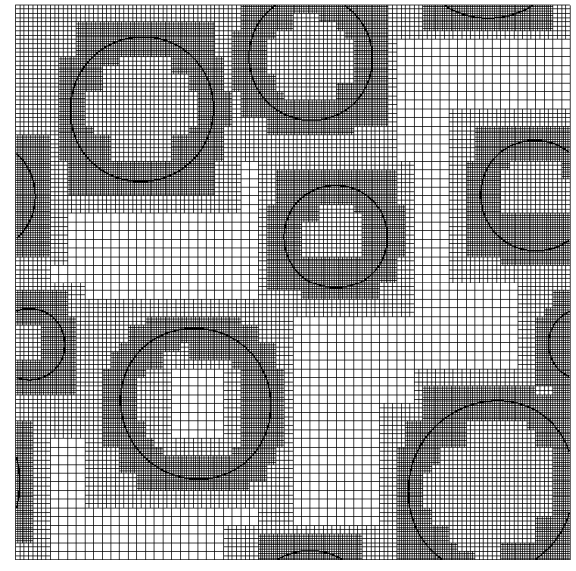

(c) $\mathrm{t}=20$

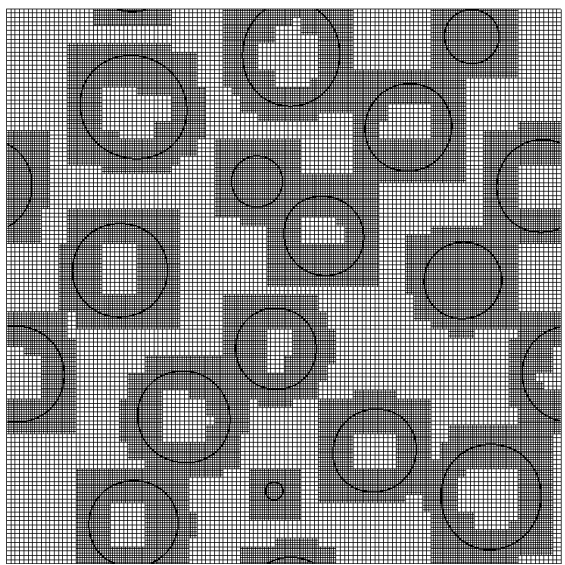

(b) $t=5$

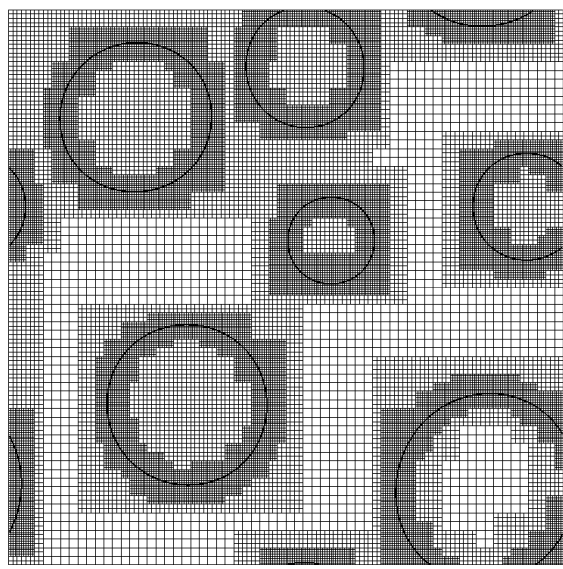

(d) $\mathrm{t}=25$

FIG. 4.14. Evolution of the concentration $c(x, y, t)$ with an average concentration $c_{\mathrm{ave}}=0.3$. The times are shown below each figure. 
For the following test, we consider spinodal decomposition using the off-critical average composition $c=0.3$. The results are shown in Fig. 4.14. The interface parameter is $\epsilon=0.0025$. The initial condition has the composition being nearly uniform with random perturbations of maximum magnitude $1 \times 10^{-3}$. The spatial domain is $1 \times 1$ and periodic boundary conditions are applied to both directions. The overall coarse computational grid is $64 \times 64$ and there are two levels of adaptivity. The AMR libraries automatically generate a grid structure that adapts to the locations of the phase interfaces. The effective fine grid resolution is $256 \times 256$. As we would expect from Cahn's theory [11], in Fig. 4.14 isolated particles form relatively quickly as the two-phase regions form, associated with $c=1$ and $c=0$, respectively. Over a much longer time scale the system coarsens, and the mesh adapts around the isolated particles. More detailed studies will be presented in a forthcoming work [32].

\section{Conclusion}

In this paper we have presented a general model of three-phase flows and developed an associated efficient, second order accurate finite difference method to solve the model equations numerically. The three-liquid phases may be fully miscible, partially miscible or immiscible. The miscibility of the phases is modeled thermodynamically through the Helmholtz free energy. An additional advantage of the model is that triple interfaces are handled without resorting to ad-hoc procedures.

We presented examples of flow with miscible and immiscible components. We demonstrated the convergence of our algorithm through a resolution study. In addition, we found good agreement with the theory for an equilibrium liquid lens (lying atop an interface). We provided demonstrations of liquid/liquid remediation. In the first example, a compound drop was simulated, in which a light fluid encapsulated a heavy contaminant drop. The light fluid causes the compound drop to rise and deposit the contaminant at an interface where it may be removed. In the second example, we investigated the diffusional transfer of a preferentially miscible contaminant from one immiscible phase to another. The transfer is enhanced by the flow and in particular the Rayleigh-Taylor instability.

In future work, we will perform more extensive studies of liquid/liquid remediation. In addition, we will investigate the complex morphologies generated from the application of chaotic mixing flow to three-phase dispersion. Under appropriate conditions, a coalescence cascade ensues and the three phases may become interpenetrating and continuous. We will also investigate the limit in which one of the phases lies on the boundary between the other phases, thus mimicking a surfactant. To perform these studies, we will incorporate adaptive block-structured mesh refinement techniques [32].

\section{Acknowledgments}

The authors acknowledge the support of the Department of Energy, Office of Basic Energy Sciences and the National Science Foundation, Division of Mathematical Sciences. The authors are also grateful for the support of the Minnesota Supercomputer Institute and the Network \& Academic Computing Services (NACS) at UCI.

\section{Appendix}

In this appendix, we present an alternative set of constitutive assumptions based on an equivalent form of the entropy expression in 2.21) different from that in 2.23). Rather than taking quantities relative to the contributions from the $N_{f}$ component, as done in $[2.23)$, we instead may consider 
quantities relative to the mean of the components. Accordingly, using the equality $\sum_{k=1}^{N_{f}} J_{k}=0$, rewrite (2.21) as

$$
\begin{aligned}
\rho \chi= & \frac{1}{T}\left(\mathbf{P}+p \mathbf{I}+\rho \sum_{k=1}^{N_{f}} \nabla c_{k} \otimes\left(\frac{\partial \mathcal{F}}{\partial \nabla c_{k}}-\frac{1}{N_{f}} \sum_{j=1}^{N_{f}} \frac{\partial \mathcal{F}}{\partial \nabla c_{j}}\right)\right): \nabla \mathbf{u} \\
& +\frac{1}{T} \sum_{k=1}^{N_{f}}\left(\mathbf{t}_{k}-\rho\left(\frac{\partial \mathcal{F}}{\partial \nabla c_{k}}-\frac{1}{N_{f}} \sum_{j=1}^{N_{f}} \frac{\partial \mathcal{F}}{\partial \nabla c_{j}}\right)\right) \cdot \nabla\left(\rho^{-1} \nabla \cdot \mathbf{J}_{k}\right) \\
& +\frac{1}{T} \sum_{k=1}^{N_{f}} \nabla\left(\mu_{k}-\frac{1}{N_{f}} \sum_{j=1}^{N_{f}} \mu_{j}\right) \cdot \mathbf{J}_{k}+\nabla \cdot\left(\mathcal{J}-\sum_{k=1}^{N_{f}} \frac{\left(\mu_{k}-\frac{1}{N_{f}} \sum_{j=1}^{N_{f}} \mu_{j}\right) J_{k}}{T}\right) .
\end{aligned}
$$

Taking the diffusion flux

$$
\mathcal{J}=\sum_{k=1}^{N_{f}}\left(\mu_{k}-\frac{1}{N_{f}} \sum_{j=1}^{N_{f}} \mu_{j}\right) \mathbf{J}_{k} / T
$$

we are now in a position to pose thermodynamically consistent constitutive relations for $\mathbf{P}, \mathbf{t}_{k}$ and $\mathbf{J}_{k}$. Arguing as in Section 2.1, we obtain the constitutive assumptions

$$
\begin{aligned}
\mathbf{P} & =-p \mathbf{I}-\rho \sum_{k=1}^{N_{f}} \nabla c_{k} \otimes\left(\frac{\partial \mathcal{F}}{\partial \nabla c_{k}}-\frac{1}{N_{f}} \sum_{j=1}^{N_{f}} \frac{\partial \mathcal{F}}{\partial \nabla c_{j}}\right)+\eta\left(\mathbf{D}-\frac{2}{3}(\nabla \cdot \mathbf{u}) \mathbf{I}\right), \\
\mathbf{t}_{k} & =\rho\left(\frac{\partial \mathcal{F}}{\partial \nabla c_{k}}-\frac{1}{N_{f}} \sum_{j=1}^{N_{f}} \frac{\partial \mathcal{F}}{\partial \nabla c_{j}}\right), \\
J_{k} & =v_{k} \nabla\left(\mu_{k}-\frac{1}{N_{f}} \sum_{j=1}^{N_{f}} \mu_{j}\right) \quad \text { for } k, \ldots, N_{f} .
\end{aligned}
$$

Using the form of the free energy from Section 2.4 , we obtain

$$
\frac{1}{N_{f}} \sum_{j=1}^{N_{f}} \frac{\partial \mathcal{F}}{\partial \nabla c_{j}}=\frac{1}{N_{f}} \sum_{j=1}^{2 N_{f}} \epsilon_{j}^{2} \nabla c_{j} .
$$

Note that if $\epsilon_{j}=\epsilon$, then the sum in 6.5 vanishes. Therefore the extra fluid stress induced by concentration gradients is the same as obtained in Section 2.4. However, the chemical potential

$$
\begin{aligned}
\tilde{\mu}_{k}=\mu_{k}-\frac{1}{N_{f}} \sum_{j=1}^{N_{f}} \mu_{j}= & \frac{\partial F}{\partial c_{k}}-\frac{1}{N_{f}} \sum_{j=1}^{N_{f}} \frac{\partial F}{\partial c_{j}}+\left(\alpha_{k}-\frac{1}{N_{f}} \sum_{j=1}^{N_{f}} \alpha_{N_{f}}\right) p \\
& -\frac{1}{2 \rho} \nabla \cdot\left(\rho \nabla\left(\epsilon_{k}^{2} c_{k}-\frac{1}{N_{f}} \sum_{j=1}^{N_{f}} \epsilon_{j}^{2} c_{j}\right)\right)
\end{aligned}
$$

is slightly different than that obtained in Section 2.4 Thus, even though 6.1 is equivalent to 2.21 and 2.23 , somewhat different constitutive relations may be taken. 


\section{REFERENCES}

1. Almgren, A. S., Bell, J. B., \& Szymczak, W. G. A numerical method for the incompressible Navier-Stokes equations based on an approximate projection. SIAM J. Sci. Comput. 17 (1996), 358-369. Zbl 0845.76055 MR 1374285

2. Anderson, D., McFadden, G. B., \& Wheeler, A. A. Diffuse interface methods in fluid mechanics. Ann. Rev. Fluid Mech. 30 (1998), 139-165. MR 1609626

3. Applied numerical algorithms group. The chombo framework for block-structured adaptive mesh refinement. Technical report, Lawrence Berkeley National Laboratory, http://seesar.lbl.gov/Chombo.

4. Barrett, J. W. \& Blowey, J. F. An improved error bound for a finite element approximation of a model for phase separation of a multi-component alloy. IMA J. Numer. Anal. 19 (1999), 147-168. Zbl 0970.65101 MR 1670685

5. Barrett, J. W. \& Blowey, J. F., \& Garcke, H. On fully practical finite element approximations of degenerate Cahn-Hilliard systems. M2AN Math. Model. Numer. Anal. 35 (2001), 713-748. Zbl 0987.35071 MR 1863277

6. Barrett, J. W. \& Blowey, J. F. An error bound for the finite element approximation of a model for phase separation of a multi-component alloy. IMA J. Numer. Anal. 16 (1996), 257-287. Zbl 0849.65069 MR 1382718

7. Barrett, J. W. \& Blowey, J. F. Finite element approximation of a model for phase separation of a multi-component alloy with nonsmooth free energy and a concentration dependent mobility matrix. Math. Models Methods Appl. Sci. 9 (1999), 627-663. Zbl 0936.65120 MR 1697389

8. Bell, J., Collela, P., \& Glaz, H. A second-order projection method for the incompressible NavierStokes equations. J. Comput. Phys. 85 (1989), 257-283. Zbl 0681.76030 MR 1029192

9. Berger, M. J. \& Oliger, J. Adaptive mesh refinement for hyperbolic partial differential equations. J. Comput. Phys. 53 (1984), 484-512. Zbl 0536.65071 MR 0739112

10. Blowey, J. F., Copetti, M. I. M., \& Elliott, C. M. The numerical analysis of a model for phase separation of a multi-component alloy. IMA J. Numer. Anal. 16 (1996), 111-139. Zbl 0857.65137 MR 1367400

11. CAHn, J. W. On spinodal decomposition. Acta Metall. 9 (1961), 795-801.

12. CAhn, J. W. \& Hilliard, J. E. Free energy of a non-uniform system. I. Interfacial free energy. J. Chem. Phys. 28 (1958), 258-267.

13. Ceniceros, H. D. The effects of surfactants on the formation and evolution of capillary waves. Phys. Fluids 15 (2003), 245-256. MR 1971799

14. Coleman, B. D. \& Noll, W. Thermodynamics of elastic materials with conduction and viscosity. Arch. Rat. Mech. Anal. 13 (1963), 167-178. Zbl 0113.17802 MR 0153153

15. COPETti, M. Numerical experiments of phase separation in ternary mixtures. Math. Comput. Simulation 52 (2000), 41-51. MR 1756463

16. Daly, B. J. Numerical study of the effect of surface tension on interface instability. Phys. Fluids 12 (1969), 1340-1354. Zbl 0177.56103

17. De Groot, S. R. \& Mazur, P. Non-Equilibrium Thermodynamics. Dover (1984) (originally published by North-Holland, Amsterdam, 1962). MR 0140332

18. Drumright-Clarke, M. A. \& Renardy, Y. The effect of insoluble surfactant at dilute concentration on drop breakup under shear with inertia. Phys. Fluids 16 (2004), 14-21.

19. Eggleton, C. D., Tsai, T.-M. \& Stebe, K. J. Tip streaming from a drop in the presence of surfactants. Phys. Rev. Lett. 87 (2001), 048302.

20. Eyre, D. J. Systems for Cahn-Hilliard equations. SIAM J. Appl. Math. 53 (1993), 1686-1712. Zbl 0853.73060 MR 1247174 
21. Garcke, H., Nestler, B., \& Stinner, B. A diffuse interface model for alloys with multiple components and phases. SIAM J. Appl. Math. 64 (2004), 775-799. Zbl pre02121942 MR 2068122

22. Garcke, H., Nestler, B., \& Stoth, B. A multiphase field concept: numerical simulations of moving phase boundaries and multiple junctions. SIAM J. Appl. Math. 60 (2000), 295-315. Zbl 0942.35095 MR 1740846

23. Hohenberg, P. C. \& Halperin, B. I. Theory of dynamic critical phenomena. Rev. Mod. Phys. 49 (1977), 435-479.

24. James, A. J. \& Lowengrub, J. A surfactant-conserving volume-of-fluid method for interfacial flows with insoluble surfactant. J. Comput. Phys. 201 (2004), 685-722. Zbl 1061.76062 MR 2100518

25. Jan, Y.-J. \& Tryggvason, G. Computational studies of contaminated bubbles. Proceedings of a Symposium on the Dynamics of Bubbles and Vorticity near Free Surfaces, I. Sahin and G. Tryggvason (eds.), ASME, 119 (1991), 46-54.

26. JiAng, G. S. \& SHU, C.-W. Efficient implementation of weighted ENO schemes. J. Comput. Phys. 126 (1996), 202-228. Zbl 0877.65065 MR 1391627

27. Johnson, R. A. \& Borhan, A. Stability of the shape of a surfactant-laden drop translating at low Reynolds number. Phys. Fluids 12 (2000), 773-784.

28. Kan, H. C., Udaykumar, H. S., Shyy, W., \& Tran-Son-Tay, R. Hydrodynamics of a compound drop with application to leukocyte modeling. Phys. Fluids 10 (1998), 760-774.

29. Kim, J. S. Ph.D. Thesis, University of Minnesota, 2002.

30. Kim, J. S., KANG, K., \& Lowengrub, J. S. Conservative multigrid methods for Cahn-Hilliard fluids. J. Comput. Phys. 193 (2004), 511-543. Zbl pre02033014 MR 2030475

31. Kim, J. S., KANG, K., \& Lowengrub, J. S. Conservative multigrid methods for ternary Cahn-Hilliard systems. Comm. Math. Sci. 2 (2004), 53-77. MR 2082819

32. Kim, J. S., Wise, S. M., \& Lowengrub, J. S. Solving Cahn-Hilliard equation using adaptive mesh refinement. In preparation.

33. Li, X. \& PozRiKidis, C. The effect of surfactants on drop deformation and on the rheology of dilute emulsions in Stokes flow. J. Fluid Mech. 341 (1997), 165-194. Zbl 0892.76013 MR 1457708

34. Lowengrub, J. S. \& Truskinovsky, L. Quasi-incompressible Cahn-Hilliard fluids and topological transitions. R. Soc. Lond. Proc. Ser. A Math. Phys. Eng. Sci. 454 (1998), 2617-2654. Zbl 0927.76007 MR 1650795

35. Milliken, W. J. \& LeAL, L. G. The influence of surfactant on the deformation and breakup of a viscous drop: the effect of surfacant solubility. J. Coll. Int. Sci. 166 (1994), 879-902.

36. Morral, J. E. \& CAhn, J. W. Spinodal Decomposition in Ternary Systems. Acta Metall. 19 (1971), 1037-1067.

37. PESkin, C. S. Numerical analysis of blood flow in the heart. J. Comput. Phys. 25 (1977), 220-252. Zbl 0403.76100 MR 0490027

38. Porter, D. A. \& Easterling, K. E. Phase Transformations in Metals and Alloys. van Nostrand Reinhold (1993).

39. Renardy, Y., Renardy, M., \& CRistini, V. A new volume-of-fluid formulation for surfactants and simulations of drop deformation under shear at a low viscosity ratio. Eur. J. Mech. B 21 (2002), 49-59. Zbl 1063.76077

40. Smith, K. A., Solis, F. J., \& Chopp, D. L. A projection method for motion of triple junctions by levels sets. Interfaces Free Bound. 4 (2002), 263-276. Zbl pre01791240 MR 1914624

41. Stone, H. A. \& Leal, L. G. The effects of surfactants on drop deformation and breakup. J. Fluid Mech. 222 (1990), 161-186. Zbl 0706.76124

42. Sussman, M., Almgren, A. S., Bell, J. B., Colella, P., Howell, L. H., \& Welcome, M. L. An adaptive level set approach for incompressible two-phase flows. J. Comput. Phys. 148 (1999), 81-124. Zbl 0930.76068 MR 1665212 
43. Sussman, M. \& Puckett, G. E. A coupled level set and volume-of-fluid method for computing 3D and axisymmetric incompressible two-phase flows. J. Comput. Phys. 162 (2000), 301-337. Zbl 0977.76071 MR 1774261

44. Trottenberg, U., Oosterlee, C., \& Schüller, A. Multigrid. Academic Press, London (2001). Zbl 0976.65106 MR 1807961

45. Truesdell, C. \& Noll, W. The Non-linear Field Theories of Mechanics. Springer, Berlin (1965). Zbl 0315.73008 MR 0193816 